\title{
Selective, modular probes for thioredoxins enabled by rational tuning of a unique disulfide structure motif
}

Jan G. Felber ${ }^{1}$, Lukas Zeisel ${ }^{1}$, Lena Poczka ${ }^{1}$, Karoline Scholzen², Sander Busker ${ }^{2}$, Martin S. Maier ${ }^{1}$, Ulrike Theisen ${ }^{3}$, Christina Brandstädter ${ }^{4}$, Katja Becker ${ }^{4}$, Elias S. J. Arnér ${ }^{2,5}$, Julia Thorn-Seshold ${ }^{1}$, Oliver Thorn-Seshold ${ }^{1, *}$

${ }^{1}$ Department of Pharmacy, Ludwig-Maximilians University Munich, Butenandtstr. 5-13, 81377 Munich, DE.

${ }^{2}$ Department of Medical Biochemistry, Karolinska Institut, Solnavägen 9, 17177 Stockholm, SE.

${ }^{3}$ Institute of Pharmacology and Toxicology, Medical Center, University of Rostock, Schillingallee 70, 18057 Rostock, DE.

${ }^{4}$ Interdisciplinary Research Centre (IFZ), Justus-Liebig University Giessen, Heinrich-Buff-Ring 26-32, 35392 Giessen, DE.

${ }^{5}$ Department of Selenoprotein Research, National Institute of Oncology, 1122 Budapest, HU.

\begin{abstract}
Specialised cellular networks of oxidoreductases coordinate the dithiol/disulfide-exchange reactions that control metabolism, protein regulation, and redox homeostasis. For probes to be selective for redox enzymes and effector proteins ( $\mathrm{nM}$ to $\mu \mathrm{M}$ concentrations), they must also be able to resist nonspecific triggering by the ca. $50 \mathrm{mM}$ background of non-catalytic cellular monothiols. However, no such selective reduction-sensing systems have yet been established. Here, we used rational structural design to independently vary thermodynamic and kinetic aspects of disulfide stability, creating a series of unusual disulfide reduction trigger units designed for stability to monothiols. We integrated the motifs into modular series of fluorogenic probes

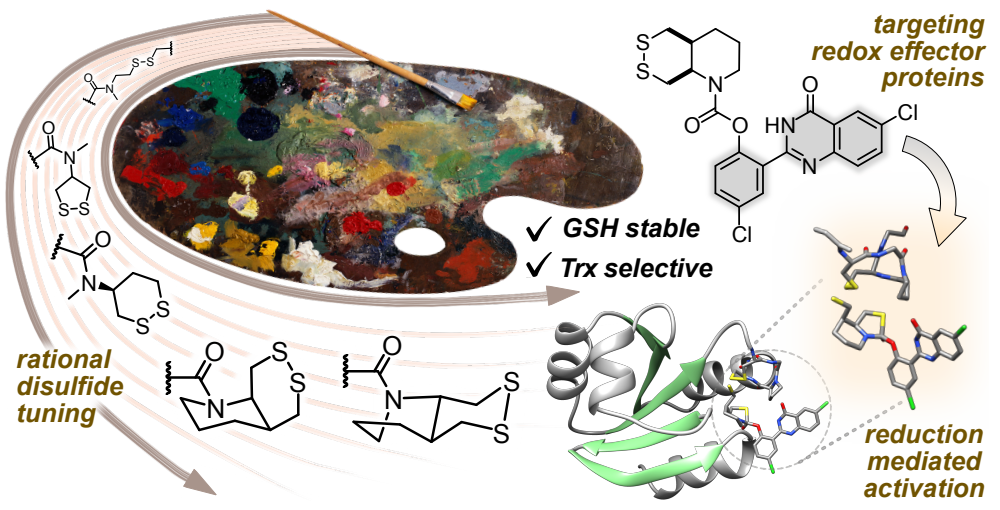
that release and activate an arbitrary chemical cargo upon reduction, and compared their performance to that of the literature-known disulfides. The probes were comprehensively screened for biological stability and selectivity against a range of redox effector proteins and enzymes. This design process delivered the first disulfide probes with excellent stability to monothiols, yet high selectivity for the key redox-active protein effector, thioredoxin. We anticipate that further applications of these novel disulfide triggers will deliver unique probes targeting cellular thioredoxins. We also anticipate that further tuning following this design paradigm will deliver redox probes for other important dithiol-manifold redox proteins, that will be useful in revealing the hitherto hidden dynamics of endogenous cellular redox systems.
\end{abstract}

\section{Introduction}

Controlled dithiol/disulfide-exchange reactions are essential to cellular metabolism, protein folding, protein regulation, and diverse aspects in cellular homeostasis and stress response. ${ }^{1,2}$ Two ubiquitous networks are particularly important: the thioredoxin $(\operatorname{Tr} x)$ - thioredoxin reductase (TrxR) system and the glutaredoxin (Grx) - glutathione (GSH) - glutathione reductase (GR) systems (Fig. 1a)., ${ }^{3,4}$ These systems are driven by catalytically active enzymes, GR and isozymes of $\operatorname{TrxR}$ (low $\mathrm{nM}$ cellular concentrations), which pass reducing equivalents from NADPH into a wide range of dithiol/disulfide-type reactions via effector proteins, especially several isozymes of Trx and Grx $(\mu \mathrm{M})$; in the case of GR, this transfer goes over the redox-active peptide GSH (mM concentration). $)^{5,6}$ The networks are interlinked, and can function as backup systems for each other. ${ }^{4,7}$ The Trx and Grx systems catalyze two-electron dithiol/disulfide-exchange reactions proceeding via polar ionic mechanisms with high substrate specificities and precise biological control. ${ }^{8,9}$ Their scope of substrates is controlled by chemocompatibility as well as protein-substrate binding, and the relative turnover rates of each step in these redox networks is tightly regulated by reaction kinetics and compartmentalization. ${ }^{10}$

The biochemistry and isoforms of the Trx and GSH/Grx networks have been excellently reviewed; ${ }^{11}$ relevant aspects are summarised here. (i) TrxRs and GRs use similar FADcontaining domains to harvest electrons from NADPH, passing them to similar structurally restricted dithiol/disulfide active sites (CVNVGC motif). ${ }^{12}$ Mammalian TrxR also has a C-terminal selenolthiol/selenenylsulfide active site (CU motif) on a flexible surface-exposed loop, to relay electrons from the NADPH-driven dithiol site onto its substrates. ${ }^{13}$ These include Trxs and related redox proteins e.g. TRP14 (also known as TXNDC17), though it can also reduce nonphysiological small molecules (Fig 1b). ${ }^{14,15}$ Due to its rare selenolthiol, TrxR has unusual redox properties compared to dithiols: with both enhanced reaction kinetics and lowered reduction potential. ${ }^{16} \mathrm{GR}$ is unusual in that it is highly specific for the small molecule glutathione disulfide (GSSG), so offering little scope for other substrates. (ii) Trxs and dithiol 
Grxs (includes Grx1 and Grx2) are thiol/disulfide redox-active proteins with surface-exposed dithiol redox-active sites $(\mathrm{CxxC}$ motifs) that recognise and reduce a variety of protein substrates in pro- and eukaryotic organisms (Fig 1b). Trxs have lower reduction potentials and are reduced by selenolthiol TrxR; Grxs have higher reduction potentials and use monothiol GSH for their reduction. ${ }^{17}$
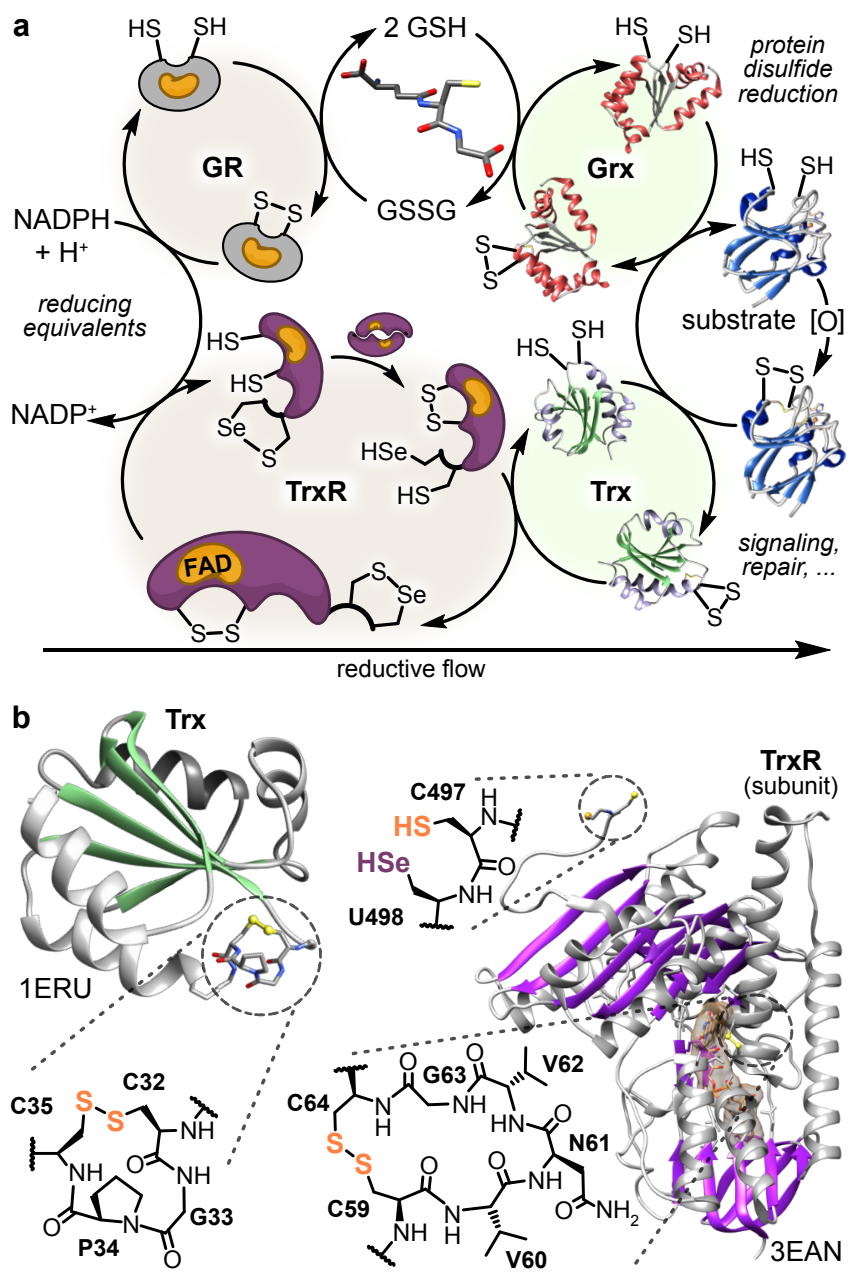

Figure 1 Key players in dithiol/disulfide homeostasis. (a) NADPH-driven cellular signal transduction and redox repair through the GSH/Grx and Trx systems. (b) Active site structures of $\operatorname{Trx}^{18}$ and $\operatorname{TrxR}^{12,14,19}$ : preorganised cyclic dichalcogenides.

In all these enzymes, the spatial preorganisation and flexibility of the dichalcogenide redox-active sites is key to their kinetics and thermodynamics (mirroring what is known for small molecule reductants). ${ }^{20}$ These factors, plus others such as protein recognition, redox potentials and non-equilibrium metabolic flows, contribute to their substrate scopes in cells though our understanding of the determinants for cellular activities of these enzymes remains poor. ${ }^{21}$

The lack of techniques to measure or respond to the turnover dynamics of these redox proteins in situ greatly limits our understanding of redox biology, because it is turnover dynamics that are key to understanding network function and homeostasis. ${ }^{2}$ So far, the best-developed probes for use in redox biology are genetically engineered ratiometric fluorescent redox-active protein sensors. ${ }^{22-24}$ These provide highly protein-specific, spatiotemporally-resolved readouts for the reduced-to-oxidised state ratio of the target redox-active proteins. However, as ratiometric readouts of redox state, they do not reveal turnover dynamics. Also, genetic approaches are monitoring-only systems that cannot respond to redox reactions with an action, such as delivering a therapeutic cargo.

To quantify turnover, selective turn-on probes are needed: whereby enzymatic turnover events can be measured by integrating cumulative and irreversible signal accumulation in the form of an activated probe. In this context, small molecule probes that employ disulfide-based trigger-cargo designs (Fig S1) are promising alternatives to protein sensors, and are potentially chemocompatible to the redox enzymes. Such probes are ideally created so that their cargo is deactivated in the oxidised state, while reduction irreversibly activates the cargo, resulting in a signal. Typically, activation proceeds by a fragmentation reaction exposing a key structural element on the cargo, often simply by cleaving it from the trigger. This is a conceptually simple design, and it is additionally attractive because unlike genetic probes, it can act upon a stimulus: modular designs can enable not only probes for detection and quantification of enzyme activity, but also prodrugs delivering drug cargos conditional upon this activity.

In the thiol redox context, trigger-cargo designs have been used to release a range of cargo types upon reduction: including phenol- and aniline-type fluorophores and drugs (Fig S1a-b). For such designs to be selective, a probe must only commit to the first irreversible step on the pathway to turnon after it reacts with the targeted enzyme - and not with any undesired species (reducing or otherwise). Therefore, while analysing thiol/disulfide-exchanges in light of equilibrium thermodynamic parameters (e.g. reduction potential $\mathrm{E}^{\circ}$ ) is generally informative, to design selective cumulative-release probes for enzyme turnover requires considering the kinetics and reversibility of each step in the reaction sequence. These, in turn, depend on the chemistry of the redox trigger and its reaction with target enzymes and reductants. For this analysis, we must distinguish the reaction of a disulfide with a single thiolate to liberate a thiolate, which we refer to as exchange, from the net reduction of a disulfide to liberate two thiolates by two stepwise exchanges, which we will refer to as reduction.

Linear disulfides are the most-used redox triggers in cargoreleasing designs (Fig S1a-b). ${ }^{25}$ However, linear disulfides cannot selectively detect or respond to enzyme activities in the cellular context. ${ }^{26-28}$ This is because thiol-disulfide exchange at either sulfur irreversibly and non-selectively commits linear disulfides to turn-on (Fig 2a); and the rapidity of even uncatalysed exchange with the high intracellular monothiol concentrations (ca. $5 \mathrm{mM} \mathrm{GSH}$, ca. $50 \mathrm{mM}$ protein thiols 
$(\mathrm{PRSH}))$, causes a high degree of monothiol-based cargo release that likely drowns out enzyme-specific components. ${ }^{26}$ Therefore, at best, linear disulfides report nonspecifically on initial thiol-disulfide exchange rates. ${ }^{29}$

Cyclic disulfide redox triggers of type A (Fig $\mathbf{2 b}$ ) instead allow kinetic reversibility in both exchange steps: their first irreversible step is the actual cargo release itself $\left(\mathrm{k}_{\mathrm{rel}}\right)$. Reversion to the cyclic disulfide $\mathbf{A}$ after exchange to $\mathbf{B} / \mathbf{D}$ $\left(\mathrm{k}_{\mathrm{el}} / \mathrm{k}_{\mathrm{g} 1}\right.$ in $\left.\mathbf{F i g} \mathbf{2 b}\right)$ may be kinetically rapid (intramolecular cyclisation), and thermodynamically favoured by enthalpic (preorganised disulfide geometry) and entropic effects (molecular cleavage). These factors can be amenable to tuning by structural design. Moreover, reversion after reduction to $\mathbf{C}$ is also well-known: it underlies the use of dithiothreitol (DTT) as a rapid, stoichiometric, nonspecific reductant of linear disulfides GSSG, $P_{R} S S G$, and $P_{R} S S P_{R}\left(k_{g_{2}}\right.$ in Fig $\left.\mathbf{2 b}\right)$. The rate $\mathrm{k}_{\mathrm{e} 2}$ of dithiol $\mathbf{C}$ reducing an enzyme vicinal disulfide may vary, depending on the enzyme, and on structural aspects of the trigger that are again amenable to rational design (indeed, nature uses such aspects to ensure that the intramolecular cyclic dichalcogenides of redox-active proteins (Fig $\mathbf{1 b}$ ) are favoured over the disulfides of their protein substrates).
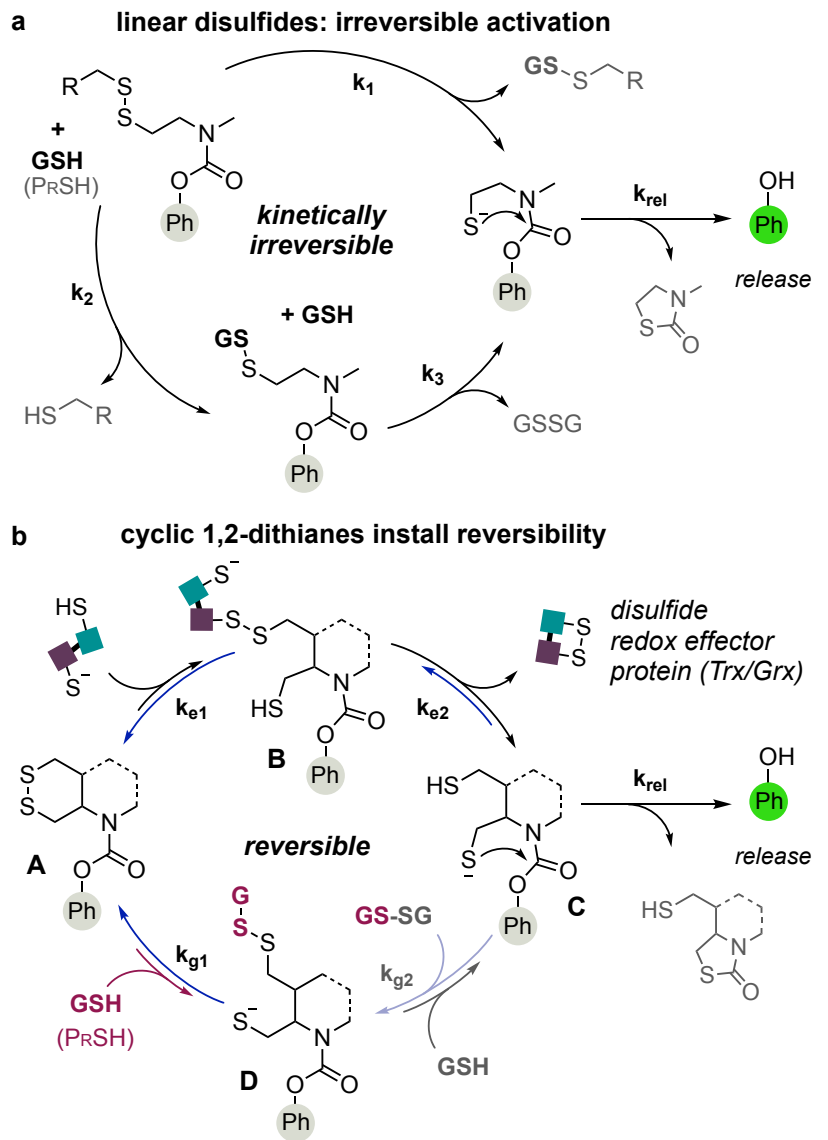

Figure 2 Mechanistic aspects of (a) irreversible monothiol reduction of linear disulfide-type reduction-sensing units; and (b) thermodynamically reversible dithiol reduction of cyclic disulfide systems; as part of irreversible release probes.
We concluded that stably preorganised cyclic disulfides A with good $\mathbf{k}_{\mathrm{g} 1}$ to resist monothiol reactivity (Fig $\mathbf{2 b}$ ) would be suitable starting points for triggers in enzyme-selective probes, and we determined to explore their synthesis and use.

Cyclic disulfide trigger substrates for trigger-cargo probes have been sparsely reported; and structures that are known have rarely been assessed for redox selectivity and kinetic performance. The most studied are strained cyclic 6membered disulfide epidithiodiketopiperazines (ETPs): but their strain-promoted reactivity with monothiols is nonspecific and significant, so they are not suitable for selective probes. ${ }^{30}$ Moderately strained 5-membered cyclic disulfides (1,2dithiolanes) had been reported to be selectively reduced by TrxR and were applied in the fluorogenic "TRFS" probes ${ }^{31-33}$ and prodrugs ${ }^{34}$. However, 1,2-dithiolanes undergo strainpromoted, kinetically irreversible, facile and nonspecific exchange with cell-surface monothiols, ${ }^{35,36}$ as known from disulfide-mediated cellular uptake studies. ${ }^{37,38}$ Our work confirmed that 1,2-dithiolane probes are, correspondingly, nonspecifically activated by monothiols. ${ }^{29}$

To resist monothiol reduction requires high reversion rates $\mathrm{k}_{\mathrm{g} 1}$ and $\mathrm{k}_{\mathrm{g} 2}$ to minimise the concentration of $\mathbf{C}$ under monothiol challenge. We thus focused on unstrained cyclic disulfides, which are in fact the most poorly explored as triggers of irreversibly-activated redox probes (Fig S1c-d; discussion in the Supporting Information). The few notable studies concern the 6-membered cyclic disulfide 1,2-dithiane. As far as we are aware, only three concepts of 1,2-dithiane designs intended to release a general molecular cargo after reduction by biological species have been disclosed. Firstly, Butora used DTT phosphoesters to release phosphate cargos after reduction, which was stated to occur by reaction with $\mathrm{GSH} ;{ }^{28,39}$ this approach was since used by e.g. Urata. ${ }^{40}$ Secondly, Xu and Tang used an aniline carbamate of DTT to release aniline cargos after reduction, which was stated to occur by reaction with $\mathrm{H}_{2} \mathrm{Se} ;{ }^{41} \mathrm{Fang}$ also applied this approach, but contradicting Butora, stated that neither TrxR nor GSH caused release. ${ }^{33}$ Thirdly, Ziv disclosed phenolic carbamate prodrugs of 4amino-1,2-dithiane, intended to release the phenol cargo after reduction, though this was only described as occurring through the "ambient reductive environment" of the cell. ${ }^{42,43}$ Relatedly, Kohn worked on 6-membered cyclic disulfide derivatives of porfiromycin and mitomycin, though not releasing a cargo; ${ }^{44,45}$ like Butora, their reports cited nonspecific dithiane exchange/reduction by monothiols (Fig S1c-d).

We consider these reports both contradictory and limited in their assessment of what cellular reductants are, or are not effective for exchange/reduction of 1,2-dithiane-based triggercargo probes: so we set out to assess this comprehensively.

We also believed that tuning rates throughout the turn-on process would prove critical for achieving protein selectivity. Particularly, we wished to explore preorganisation by annelating the cyclic disulfide triggers (dotted ring in Fig $\mathbf{2 b}$ ). 
This modification adds minimal steric pressure at the redoxactive -SS- site, so it might not suppress enzyme docking, but it could bring two favourable effects into play: (i) increasing thiol cyclisation rate of $\mathbf{C}\left(\mathrm{k}_{\mathrm{rel}}\right)$, to accelerate signal generation without changing the reductant selectivity profile, and so improve probe performance (NB: $\mathrm{k}_{\text {rel }}$ equally increased by both cis- and trans-annelation); (ii) stabilising the cyclic disulfide $\mathbf{A}$, to increase $\mathrm{k}_{\mathrm{e} 1}$ and $\mathrm{kg}_{\mathrm{g} 1}$ and so potentially change the selectivity profile (NB: these effects should depend on the cis-/transstereochemistry). Such annelated disulfides have not yet been reported, so we wished to develop scalable and convenient syntheses, enabling their general application to redox research.

Lastly, we noted that according to our proposed mechanism, as long as hydrolytic cargo release is blocked, the choice of cargo only influences $\mathrm{k}_{\mathrm{rel}}$ and should not affect a probe's reductant selectivity. We were therefore curious to use the same trigger with different cargos, to test the bioreductant selectivities, and to rationally tune probe performance.

In this work we address the lack of information on cyclic disulfide suitability for probes. We test our design principles, by stepwise exploration of the features predicted to determine cargo release selectivity and rate. We scalably synthesise novel cyclic disulfide-based triggers, and apply them in robust, irreversible-release probes suitable for generalised cargos. We comprehensively evaluate the probes' selectivity for key redox- active proteins, and their resistance to a high monothiol background, also benchmarking previously reported but poorly-characterised disulfides. This will reveal the potential of preorganised disulfides as enzyme-selective reduction sensors, and identify a promising Trx-selective probe design.

\section{Results and Discussion}

2.1 Trigger Design. We designed a broad panel of disulfide-based redox triggers for this study (Fig 3a). To avoid unspecific monothiol-triggered cargo release, we used stable cyclic 6-membered disulfide triggers: most simply, disulfide trigger SS60. To study how preorganising annelation affects both cargo release and selectivity profiles, we designed cis- and trans-fused triggers SS66C and SS66T. As linear disulfide controls, we used simple trigger $\mathbf{S S O 0}^{25}$ and annelated SSO6 (which, when compared, should test for preorganisation only of cargo release $\mathrm{k}_{\mathrm{rel}}$ ). Where needed, we compare the results with those of the strained and/or polymerisable monocyclic disulfide controls SS50 ${ }^{29,31}$ and $\mathbf{S S} 7^{46}$. This broad panel of disulfide structure motifs would allow us to compare reductant selectivity and cargo release rates, across stable or preorganised or strained, cyclic or linear disulfide redox probes, on a level that to our knowledge has not yet been attempted. Lastly, we introduced $\mathbf{0 5 6}$ as a similarly polar but non-reducible negative control trigger, to check stringently for stability against nonreductive chemical or enzymatic release of probe cargos. a
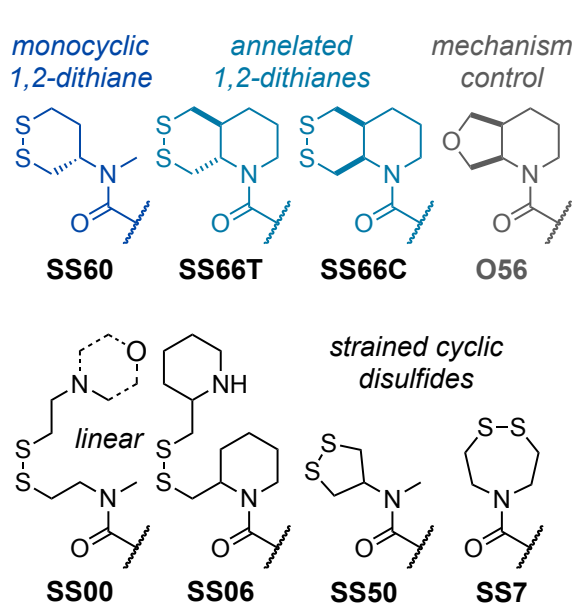
disulfides
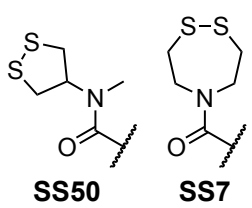

b unmaskable cargos

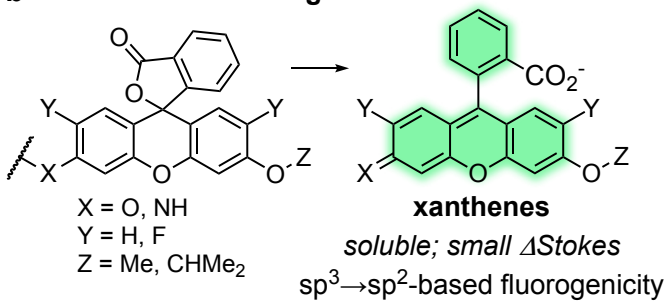

$\mathrm{sp}^{3} \rightarrow \mathrm{sp}^{2}$-based fluorogenicity

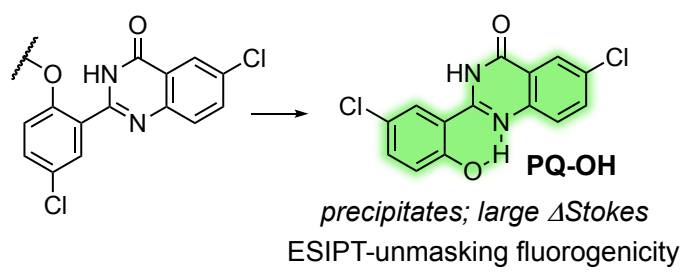

C representative probes
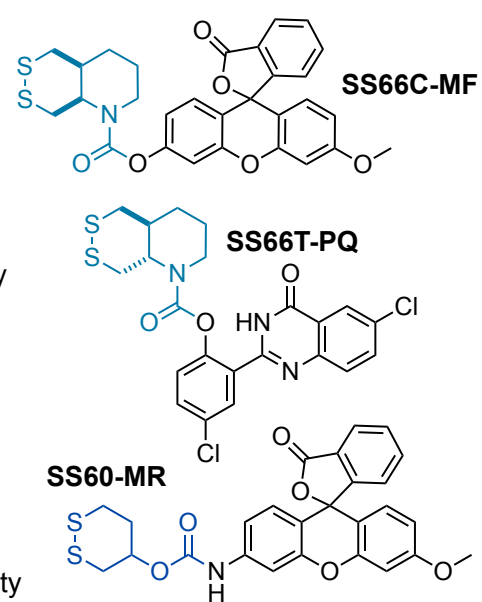

Figure 3 Trigger-and-cargo design for redox probes. (a) Stable cyclic disulfide-based reduction sensors, and their control and comparison systems. (b) Complete-off-to-on fluorogenic cargos based on phenol mono-unmasking. (c) Representatives of the 20 fluorogenic probes used in this study, derived by trigger-cargo combinations.

2.2 Probe design considerations. To create modular probe designs, we chose to work primarily with phenolic fluorophores. Anilines are simpler to adapt into fluorogenic probes, as acylation usually substantially suppresses fluorescence (though only in special cases does it do so completely), and since acylated anilines have good hydrolytic stability $^{33}$. Aniline carbamates are well-represented in triggerprobe designs. However, the poor leaving-group nature of anilides results in slow cargo release for 5-exo-trig thiol cyclisation, with physiological halflives of $>3 \mathrm{~h}$ even under fully reduced conditions. ${ }^{33}$ Alternative anilide designs such as amides and ureas are not cleavable and so do not represent modular response systems as we sought. ${ }^{33,47}$

By comparison, phenol-releasing systems could benefit from superior leaving-group nature to improve release kinetics. Several phenolic systems featuring true off-to-on 
fluorogenicity are known; and phenols are found as key activity-determining groups in a broad range of diagnostic or therapeutic cargos, meaning that modular designs in this work could be extended into a useful series of probes and prodrugs. Stability against non-reductive hydrolysis is the major challenge for most phenols. For example, disulfide-trigger phenolic esters and carbonates have been reported ${ }^{48,49}$ but non-reductive phenol release by spontaneous and enzymatic hydrolysis is certain to prevent them achieving any selectivity in biological settings. Phenolic carbamates have previously been reported to be unstable..$^{33}$ However, we interpreted this instability as the spontaneous $\mathrm{E}_{\mathrm{1cB}}$ elimination pathway that affects primary carbamates, ${ }^{50}$ so we designed all triggers for secondary carbamate linkages to avoid this (Fig 3a),,$^{51,52}$ guided by precepts from our earlier work. ${ }^{29,53}$

2.3 Cargo Choice and Use. We selected two major series of fluorescent cargos to be masked by mono-O-carbamylation (Fig $3 \mathbf{b}$ ), requiring that this should entirely suppress their fluorescence by mechanism-based quenching. (i) We used xanthene-based mono-O-alkylated fluorescein $\mathbf{M F}-\mathbf{O H}^{54}$ and its acidified photostable congener IG-OH ${ }^{55}$ (as its $i \mathrm{Pr}$ might resist nucleophilic attack better than MF-OH's methyl). Their mono-O-carbamylation mechanistically quenches fluorescence by locking the central carbon as $\mathrm{sp}^{3}$, so destroying conjugation. Conjugation is restored by cleaving the carbamate substrate (simplifying kinetics compared to typical double-substrate probes such as fluorescein diacetate), giving soluble, biocompatible, bright fluorophores (ex/em $485 / 515 \mathrm{~nm}$ ). (ii) We also used the precipitating fluorophore PQ-OH, ${ }^{56,57}$ whose high-quantum-yield, large-Stokes-shift fluorescence (ex/em 360/520 nm) depends on excited state intramolecular proton transfer (ESIPT) of the phenolic hydrogen. Carbamylating the phenol entirely and mechanistically abolishes ESIPT fluorescence of the probe. The electron-withdrawn $\mathbf{P Q}$ should also have fast release. To compare our phenolic designs to prior art in aniline disulfideredox probes, we also prepared 3-O-methyl-rhodol MR- $\mathbf{N H}_{\mathbf{2}}$ for kinetic comparisons. ${ }^{58}$ Thus, all our probes feature a true off-to-on mechanistic switch of signal upon disulfide trigger cleavage, thereby maximising their signal-to-noise ratio and simplifying analysis (details in Fig S2).

We combined triggers freely with cargos to create a panel of probes for analysis. We name the resulting probes by the combination used; e.g. in Fig 3c, SS66C-MF is a SS66C-trigger MF-OH-releasing probe, etc. (further details and discussion in Supporting Information).

2.4 Diastereomerically pure synthesis of annelated SS66 disulfides. The simpler disulfide redox triggers were accessed straightforwardly. Monocyclic disulfide SS60 was obtained using the approach reported by Raines ${ }^{59}$ to give the protected primary amine, then by $N$-methylation and deprotection to prepare the novel secondary amine SS60. Known polymerisation-prone ${ }^{36,60}$ unstable cyclic disulfides SS50 and SS7 were accessed from aminopropanediol ${ }^{29}$ and from a nitrogen mustard precursor $^{46}$ respectively, as described. Symmetric linear disulfides SSO0 and SSO6 were accessed by oxidative dimerization of the respective thiols; as far as we know, cyclisation-preorganised linear disulfides like SS06 have not been studied before (Fig 3a). The secondary amine of these symmetric disulfides proved inconvenient for yields and purification, with double-reaction side products being formed during later carbamate coupling. Unsymmetric linear disulfides of the SSO0-type are rarely reported in the literature, and we found no convenient methods towards them. We developed a versatile batch synthesis strategy for unsymmetric linear disulfides by coupling easily-prepared, isolable precursors (one primary $S$-acetyl and one primary $S$-tosyl), achieving high chemoselectivity, and isolating the unsymmetric morpholino-substituted SSO0M (Fig 3a) in good yields. For details, see the Supporting Information.

The bicyclic redox triggers were more challenging (Fig 4). We first focused on the cis-fused SS66C system, setting the stereochemistry by heterogeneous hydrogenation of pyridine $\mathbf{1}$. Boc-protection to amine $\mathbf{2}$ then ester reduction gave cis-diol 3, which we could not however convert into a bicyclic disulfide. During Mitsunobu reaction with thioacetic acid under Raines' conditions, ${ }^{59}$ no bis(thioacetate) but rather undesired cis-ether $\mathbf{5}$ was isolated, with $\mathrm{X}$-ray confirming its structure. Fortunately, $\mathbf{5}$ provided ideal non-reducible hydrolysis controls, as the $\mathbf{0 - 5 6}$ series. Other strategies to activate the hydroxy groups for S-nucleophile substitution were unsuccessful; e.g., attempted bis(sulfonylation)s gave intramolecularly cyclised carbamates e.g. 4, as known for Boc-/Cbz-methanolpiperidines. ${ }^{61-63}$ To avoid carbamate cyclisations, we switched to $\mathrm{PMB}$ as the $\mathrm{N}$-protecting group. Diastereomerically pure $N$-PMB-diol 10 was accessed in decent yield by hydrogenation of $\mathbf{1}$, ester reduction to $\mathbf{9}$, and $\mathrm{N}$-alkylation. It was crucial to reduce the esters before nitrogen protection, to avoid partial cis-trans-isomerisation at the diester stage. Mesylation of diol $\mathbf{1 0}$ and nucleophilic substitution with KSAc gave bis(thioacetate) 11, and tandem basic acetetate cleavage/oxidation gave novel annelated disulfide 12. $\mathrm{PMB}$ was deprotected without reductive or oxidative conditions by using 2-chloroethyl chloroformate (ACE-Cl) ${ }^{64}$ giving the precursor to all SS66C probes (Fig 4).

For the trans-fused SS66T, we took a route exploiting the previous setbacks to our advantage. First, we could use basic cis-trans-epimerisation to recycle cis-diester $\mathbf{2}$, after some trials reaching an inseparable 2:1 trans:cis epimerised mixture by $\mathrm{NaOMe}$ treatment then quenching by $\mathrm{AcOH}$. Ester reduction gave 2:1 cis:trans-diol 6, still unseparated. Then, we could selectively destroy the residual cis while carrying the trans through its next step of thio-Mitsunobu reaction: trans-diol 6 converted smoothly to the trans-bis(thioacetate) 7 , while cisdiol 6 converted to cis-ether 5 , separable by chromatography. Thioester hydrolysis of 7, dithiol oxidation, and deprotection gave diastereomerically pure trans-8, the key SS66T precursor, in decent yields (Fig 4). (This also suggested that 
the cis-fused system's greater tendency to 5-exo-tet cyclisation than the trans, might be reflected in a greater kinetic and/or thermodynamic stability of its cis-disulfide: which we soon examined, as described below).

Deprotected secondary amines of the disulfide triggers were reacted with $O$-chloroformates of phenolic fluorophores to deliver 19 fluorogenic MF, IG and PQ series probes (Fig 4) and one MR-reference compound. These were all colourless and nonfluorescent both as solids and in solution, as expected for their mechanistically-quenched emission. To assess disulfide thermodynamics, we also benzoylated the amines to give "SS-Bz" compounds that mimic the geometry of the triggers in the carbamate probes, but can be reversibly equilibrated as well as crystallised (see below).

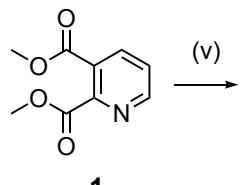

1

2

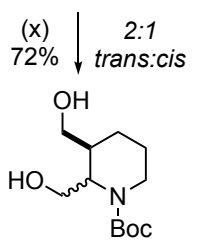

6

(xi) $\downarrow \begin{aligned} & \text { trans-selective } \\ & \text { thio-Mitsunobu }\end{aligned}$

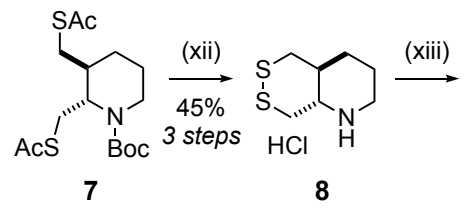

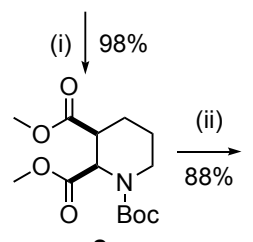

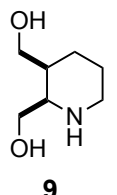

9

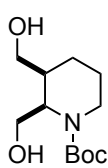

3

(iv) $\downarrow 90 \%$

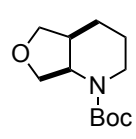

5

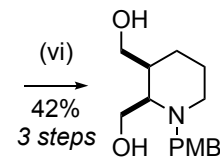

10

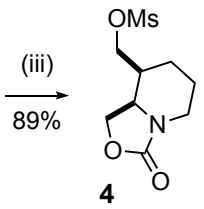

4

(viii)

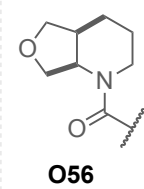

056

control

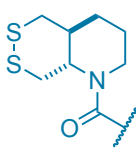

SS66T

probes
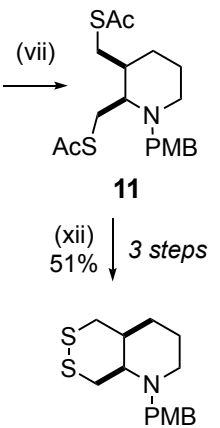

12

(ix)

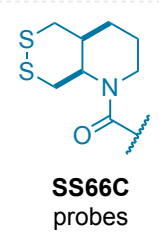

+5 other

trigger types

total: probes
20 fluorogenic

Figure 4 Diastereomerically pure syntheses of SS-66T- and SS-66C-series bicyclic disulfide redox probes. (i) 1) $\mathrm{H}_{2}, \mathrm{H}_{2} \mathrm{SO}_{4}$, $\mathrm{Pd} / \mathrm{C}, \mathrm{MeOH}, 80$ bar, r.t., 15 h; 2) $\mathrm{Boc}_{2} \mathrm{O}, \mathrm{NEt}_{3}$, dioxane $/ \mathrm{H}_{2} \mathrm{O}$, r.t., $15 \mathrm{~h}$ (98\%). (ii) $\mathrm{LiAlH}_{4}, \mathrm{Et}_{2} \mathrm{O}, 0{ }^{\circ} \mathrm{C}$ to r.t., 3 h. (88\%). (iii) $\mathrm{MsCl}$, $\mathrm{DCM}, \mathrm{NEt}_{3}$, r.t., $15 \mathrm{~h}(89 \%)$. (iv) cis-selective tetrahydrofuran formation: $\mathrm{PPh}_{3}, \mathrm{DIAD}$, (HSAc,) THF, r.t., $15 \mathrm{~h}$ (90\%). (2) $\mathrm{HCl}$, $\mathrm{MeOH}, 80^{\circ} \mathrm{C}, 4 \mathrm{~h}$ (quant.). (v) 1) $\mathrm{H}_{2}, \mathrm{H}_{2} \mathrm{SO}_{4}, \mathrm{Pd} / \mathrm{C}, \mathrm{MeOH}$, 80 bar, r.t., 15 h; 2) $\mathrm{LiAlH}_{4}, \mathrm{THF}, 0{ }^{\circ} \mathrm{C}$ to r.t., $3 \mathrm{~h}$. (vi) $\mathrm{PMB}-\mathrm{Cl}$, $\mathrm{K}_{2} \mathrm{CO}_{3}, \mathrm{EtOH}$, r.t., $15 \mathrm{~h}$ (v-vi: $42 \%$ over 3 steps). (vii) 1 ) $\mathrm{MsCl}$, $\mathrm{NEt}_{3}$, DCM, r.t., 1 h; 2) KSAc, DMF, r.t., 15 h. (viii) $\mathrm{KOH}, \mathrm{MeOH}$, r.t., 15 h; (vii-viii: $51 \%$ over 3 steps) (ix) 1) ACE-Cl, DCE, $80^{\circ} \mathrm{C}$, $5 \mathrm{~h} ; 2) \mathrm{MeOH}, 80^{\circ} \mathrm{C}, 2 \mathrm{~h}$; 3 ) carbamate coupling. (x) 1) $\mathrm{NaOMe}$, $\mathrm{MeOH}$, r.t., 1 h; then $\mathrm{AcOH}(2: 1$ trans:cis $) ; 2) \mathrm{LiAlH}_{4}, \mathrm{Et}_{2} \mathrm{O}, 0^{\circ} \mathrm{C}$ to r.t., $2 \mathrm{~h}$. (72\% over 2 steps) (xi) trans-selective bis(thioacetylation): $\mathrm{HSAc}, \mathrm{PPh}_{3}$, DIAD, THF, r.t., 15 h. (xii) 1) $\mathrm{KOH}, \mathrm{MeOH}$, r.t., 15 h; 2) $\mathrm{HCl}$, r.t., $4 \mathrm{~h}$ (xi-xii: $45 \%$ over 3 steps). (xiii) carbamate coupling.
2.5 Thermodynamic analyses: redox potentials are lowest for annelated cyclic disulfides (SS66). Redox potentials depend on structural/geometric features that (de)stabilise the disulfide relative to the dithiol. Bond distance $\Delta(\mathrm{SS})$, bond angles $\theta(\mathrm{CSS} / \mathrm{SSC})$, and dihedral angle $\varphi(\mathrm{CSSC})^{29}$, together with strain factors, typically affect disulfide stability. We could acquire X-ray structures of all three SS-Bz 1,2-dithianes (Fig 5a), showing their disulfide rings in chair-like conformations with minimal eclipsing, and CSSC dihedral angles close to the model 1,2-dithiane DTT ${ }^{\text {ox }}$ $\left(56^{\circ}\right)^{65}$. But, while a double-chair conformation was found for SS66C-Bz (similar to a cis-decalin), against our expectations SS66T-Bz formed a more strained boat-chair system unlike a trans-decalin (for further discussion see Raines ${ }^{66}$ and references therein, and Supporting Information).

We then experimentally examined the thermodynamic reduction potential $\mathrm{E}^{\circ}$ of the cyclic disulfides. We had anticipated that thermodynamic stability (low $\mathrm{E}^{\circ}$ ) would be important to resist reduction by monothiols and so to achieve selectivity for dithiol species. Whitesides calculated and experimentally determined $\mathrm{E}^{\circ} \mathrm{s}$ of uncommon disulfides in the 1980s-90s. ${ }^{60,67}$ The lowest $\mathrm{E}^{\circ}$ they reported were indeed for 2,3-dithiadecalin-type disulfides, ${ }^{68}$ which under nonstandard conditions (50:50 buffer:cosolvent) were $-0.35 \mathrm{~V}$ (trans) and $-0.34 \mathrm{~V}$ (cis). This extraordinary stability compared to linear disulfides $\left(\mathrm{E}^{\circ} \mathrm{ca} .-0.22 \mathrm{~V}\right)^{60}$ was promising for our aims in this study. However, while we expected the $\mathrm{E}^{\circ}$ of the tetrahedral amine triggers SS66T and SS66C to match these, we believed that the restrained geometries of the carbamates would modify the probes' potentials. To mimic these while avoiding the influence of kinetic considerations that also determine irreversible probe performance (trigger reduction and thiol cyclisation rates), we again used the reversible SSBz series; these also allowed us to benchmark our reduction potentials under normalized conditions.

To determine reduction potentials, we equilibrated the SS-Bz series against chemocompatible reductant standards, analysing oxidised:reduced compound ratios by HPLC. We did this similarly to methods used by Raines ${ }^{59}$ and others ${ }^{60,69}$ though with three additional features. Firstly, the benzamide is a convenient UV-active tag for HPLC signal integration. Secondly, we detected oxidised:reduced ratios for both benzamide probe and reductant standard (e.g. DTT); this controls for parasitic oxidation by adventitious oxygen (which would be reflected by an increasing total of oxidised species). Thirdly, we performed timecourse measurements to follow the progress of equilibration, and upon reaching stable plateaus we controlled for reversibility by re-oxidising all dithiols to disulfides using $\mathrm{I}_{2}$ (Fig $\mathbf{5 b}$ ); this controls for polymerisation of strained disulfides which risks being misinterpreted. By titrating reductants, we determined reduction potentials of $-0.276 \mathrm{~V}$ for $\mathbf{S S 6 0 - B z},-0.317 \mathrm{~V}$ for SS66T-Bz, and -0.339 V for SS66C-Bz (Fig 5c-d); whereas the strained analogues SS50-Bz and SS7-Bz were not 
equilibrating systems. We estimate $\pm 0.01 \mathrm{~V}$ precision in our experiment. The lower reduction potentials of the annelated SS66 compared to the monocyclic SS60 matched expectations. Potentially due to the benzamide strain,

a

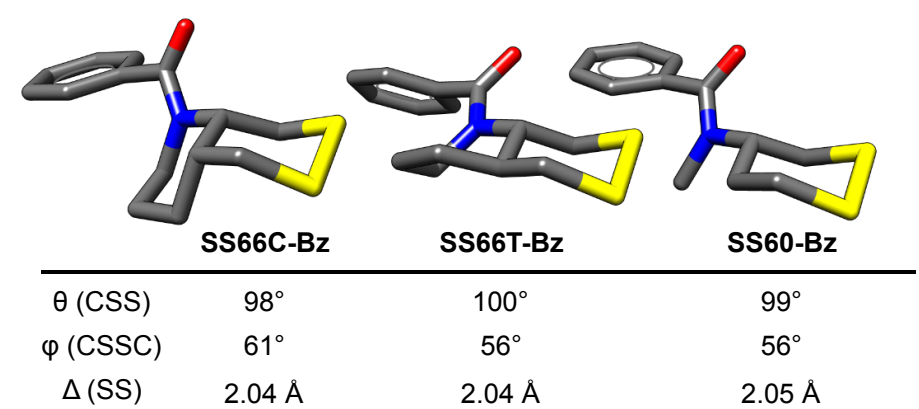

C

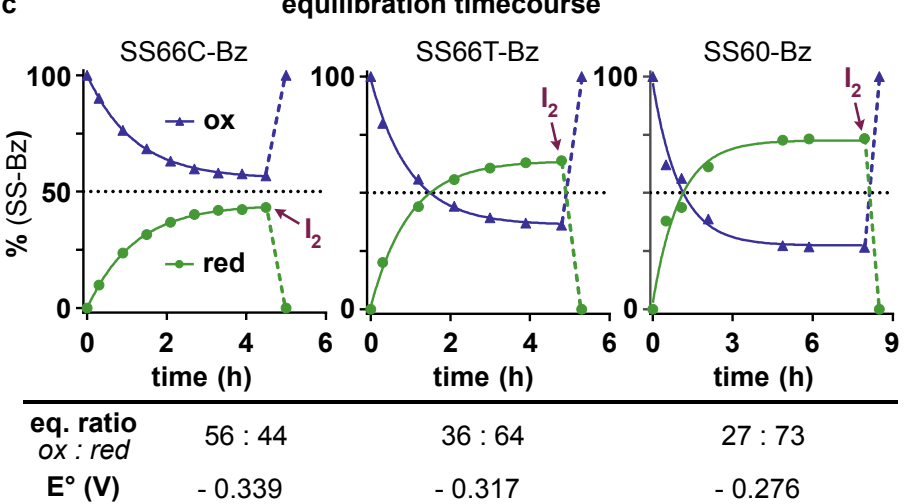

SS66T-Bz indeed had 0.02 V higher $\mathrm{E}^{\circ}$ than SS66T-Bz; while Whitesides gives a $0.01 \mathrm{~V}$ lower potential for the trans-2,3dithiadecalin $^{60}$ this may only hold true for the SS66 amines (Fig 5d; see also Supporting Information).

b

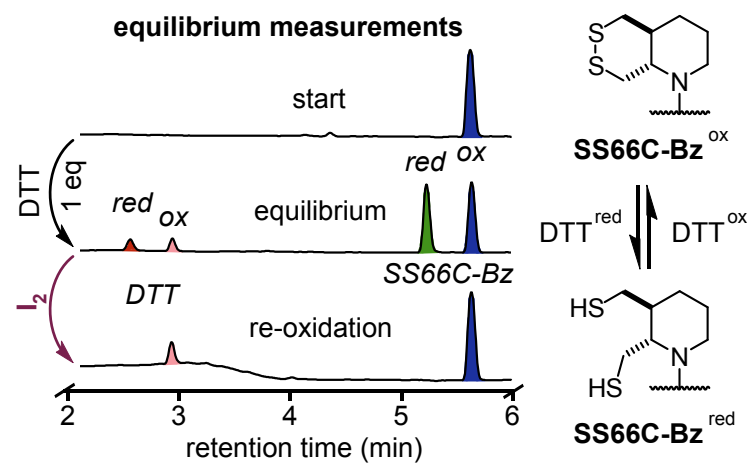

d

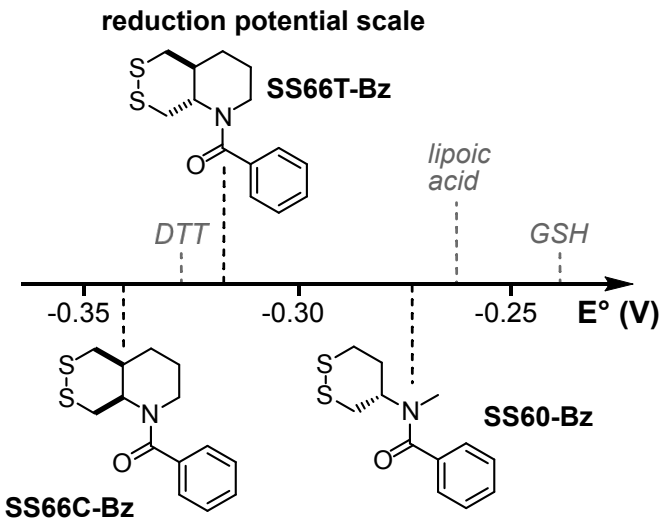

Figure 5 Redox thermodynamics of cyclic six-membered disulfide triggers. (a) single crystal X-Ray structures of 1,2-dithianes: CSS bond angles $\theta$; CSSC torsion angles $\psi$; disulfide bond distance $\Delta$. (b) HPLC-based $\mathrm{E}^{\circ}$ measurement protocol. Disulfides are equilibrated against $\mathrm{DTT}^{\text {red }}$, then dithiols are quantitatively re-oxidised by iodine as a control. (c) HPLC timecourses, equilibrium ratios, and resulting disulfide $\mathrm{E}^{\circ}$ values. (d) Graphical depiction of $\mathrm{E}^{\circ}$ for this study's 1,2-dithianes against known (bio-)reductants. ${ }^{70}$

2.6 Kinetic analyses: cyclic 6-membered disulfides resist GSH reduction, but are triggered by vicinal dithiols. We now turned to the PQ- and MF/IG-based fluorogenic probes, to test their activation by, or resistance to, small molecule reductants.

Calibrations on the cargos MF-OH, IG-OH and PQ-OH confirmed large linear ranges $(20 \mathrm{nM}-30 \mu \mathrm{M}$ for xanthenes, $>1 \mu \mathrm{M}$ for PQ-OH; Fig S2a-b). Probe calibrations and exemplary proof of the fluorogenic character highlighted their true off-to-on fluorescence switching, based on complete mechanistic quenching in the intact probes (FigS2c). Throughout the reductant challenge assays, we controlled against fluorophore release by non-reductive degradation mechanisms (such as carbamate hydrolysis, or carbamate thiolysis by thiol reductants) using the $\mathbf{0 5 6}$ probe series. Matching our design requirements, the $\mathbf{0 5 6}$ series showed in all cases that the secondary amine carbamates were entirely robust, thereby solving the problems with prior art phenolic carbamates $^{33}$ and indicating that disulfide reduction and intramolecular thiol cyclisation would be the only pathway for signal generation with our disulfide probe series.
First, to report selectively on enzyme activity, a probe must resist reduction by the cellular monothiol background (ca. $50 \mathrm{mM}$, of which up to $5 \mathrm{mM} \mathrm{GSH}) .{ }^{71}$ We therefore began by incubating probes with monothiols $(1 \mathrm{mM})$ to profile their monothiol resistance. Linear SS00- and SS06probes, and strained 5-membered cyclic SS50-probes, were rapidly triggered by all monothiols: GSH, cysteine, cysteamine (CA), N,N-dimethyl cysteamine (MEDA) and $N$ acetylcysteine (NAC). In contrast, 1,2-dithiane SS60-, SS66C- and SS66T- as well as 7-membered cyclic SS7-probes resisted monothiol reduction (Fig 6a, Fig S4-S5 for PQseries, Fig S6 for MF-series, Fig S7 for IG- and MR-series).

Next, we examined the kinetic performance of each probe, using the rapid and quantitative disulfide reducing agent tris(2-carboxyethyl)phosphine (TCEP). In these settings where no dithiol re-oxidation is possible (cf. $\mathrm{k}_{\mathrm{e} 2}$ and $\mathrm{k}_{\mathrm{g} 2}$, Fig 2), probe signal generation depends on the rates of reduction by the phosphine, 5-exo-trig thiol cyclisation, and cargo expulsion. Under such special conditions, the cargo nature rather than the disulfide structure became the major determinant of signal kinetics (Fig 6b; PQ-OH release approx. 10 times faster than MF-OH). While some reports 
claim that distal cargos substantially affect the reductant selectivity profile ${ }^{72}$, we attribute the faster release of $\mathbf{P Q}-\mathbf{O H}$ than MF-OH simply to its better leaving-group character.

Pleasingly, the relative rates of probes within each series were almost identical for the MF as for the $\mathbf{P Q}$ series (Fig $\mathbf{6 b}$; fits in Fig S3), revealing trigger-based performance features. (1) While annelations have been used for amine cyclisation promotion $^{53,73}$, this has not yet been studied for thiols. The ca. 3 -fold rate enhancement of annelation in the linear disulfides was significant (SS06 > SS00: also suggesting that their thiol cyclisation is more rate-determining than their phosphine reduction). Annelation in the 1,2-dithianes was interesting: while the cis-fused SS66C was faster than SS60 as expected, the trans-fused SS66T was slower than SS60. (2) Thiol cyclisation/cargo elimination ( $\mathbf{C} \rightarrow \mathrm{PhOH}$, Fig $\mathbf{2})$ occurs on a scale of minutes. As intramolecular thiol-disulfide exchanges $(\mathbf{B} \rightarrow \mathbf{A}, \mathbf{B} \rightarrow \mathbf{C}$ and $\mathbf{D} \rightarrow \mathbf{A})$ typically take $<10 \mathrm{~ms},{ }^{74}$ we propose that "on-reductant" cyclisation of the exchange intermediates $\mathbf{B}$ and $\mathbf{D}$ to release $\mathrm{PhOH}$ before their full reduction to $\mathbf{C}$ (Fig 2) is not a major contributor to cargo release. (3) The rates of bisthiolate cyclisation (from SS50) were twice those of the correponding monothiolate (SSO0), a satisfying match to theory; though the SS7 probes were sluggish and behaved irreproducibly. (We believe oligomerisation of $\mathbf{S S} 7^{35,60,67}$ causes its poor performance, paralleling the behaviour of polymerisable ${ }^{29} \mathbf{S S 5 0}$ probes. Additionally, neither SS7-Bz nor SS50-Bz performed reliably in the equilibration/reoxidation assay. So, while we carried these probes through all assays, we focus on interpreting only the reliable 1,2-dithiane and linear disulfide results; see Supporting Information.) a

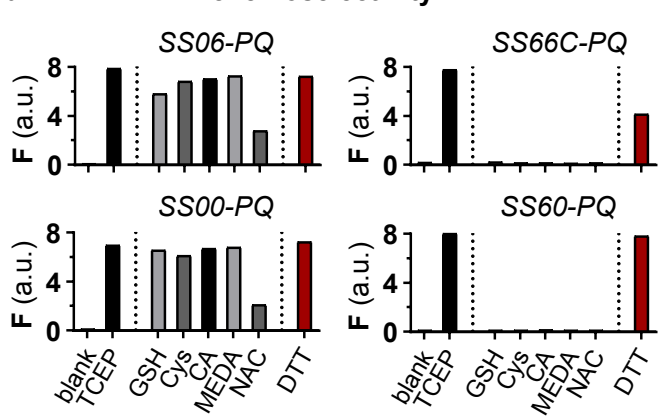

b activation kinetics - TCEP

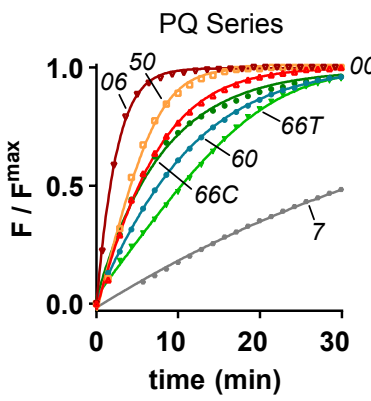

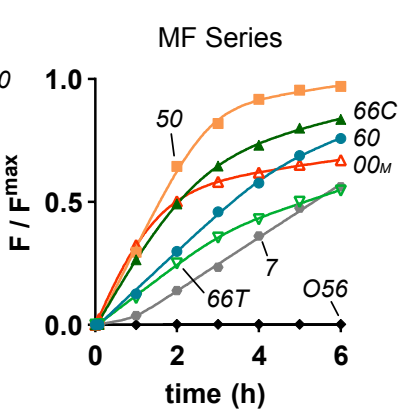

relative kinetics

\begin{tabular}{c|c|c} 
& $\mathrm{PQ}$ & $\mathrm{MF}$ \\
\hline $\mathbf{0 0}_{(\mathbf{M})}$ & 1.4 & 1.1 \\
$\mathbf{0 6}$ & 4.0 & n.d. \\
$\mathbf{6 0}$ & 1 & 1 \\
$\mathbf{6 6 C}$ & 1.4 & 1.6 \\
$\mathbf{6 6 T}$ & 0.7 & 0.8 \\
$\mathbf{5 0}$ & 2.3 & 2.2 \\
$\mathbf{7}$ & 0.2 & 0.5
\end{tabular}

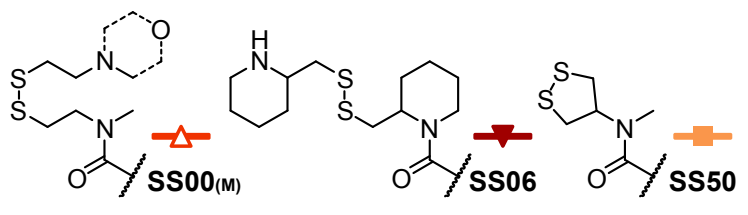

C

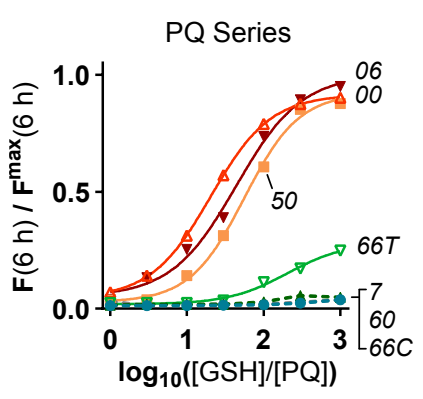

MF Series

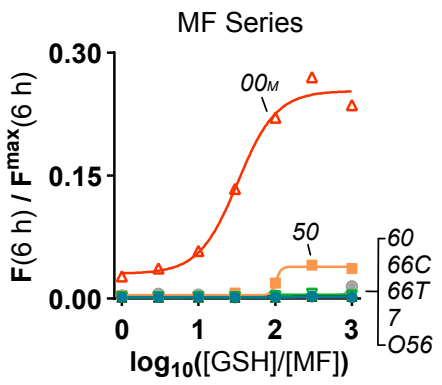

d

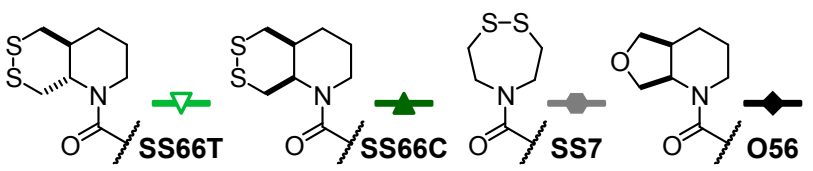

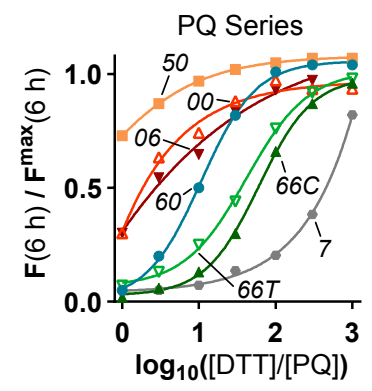

SS60-

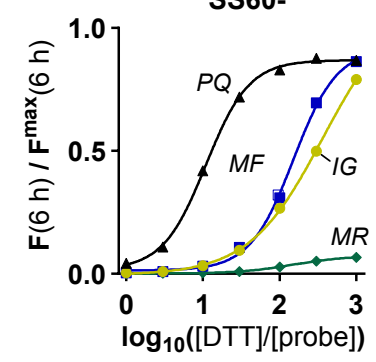

dithiol response - DTT

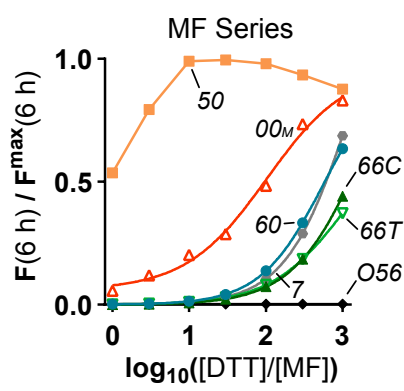<smiles>CC(C)Oc1ccc(Cl)cc1-c1nc2ccc(Cl)cc2c(=O)[nH]1</smiles>

PQ-Series<smiles>[Z]Oc1cc2c(cc1[X])C1(OC2)OC(=O)c2ccccc21</smiles>

MF: $Y=\mathrm{H}, \mathrm{Z}=\mathrm{Me}$

IG: $Y=F, Z=\mathrm{CHMe}_{2}$

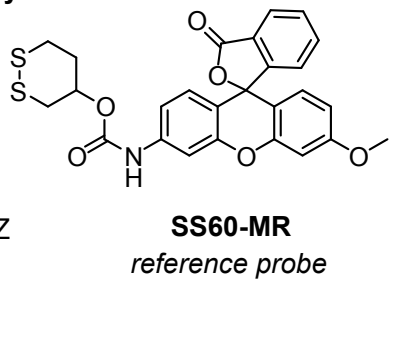

SS66C-

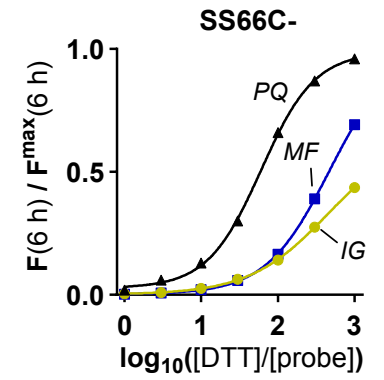

Figure 6 Reductant-resistance profiling and cargo-release kinetics. (a) Fluorescence response of representative linear and 1,2-dithiane probes to monothiol reductants $(1 \mathrm{mM})$, benchmarked to quantitative reductant TCEP $(100 \mu \mathrm{M})$ and to dithiol DTT $(1 \mathrm{mM})$; $\mathrm{t}=2 \mathrm{~h}$. (b) Probe activation kinetics with TCEP $(100 \mu \mathrm{M})$. (c) GSH challenge titrations (0.01-10 mM GSH, $10 \mu \mathrm{M}$ probe, $\mathrm{t}=6 \mathrm{~h}$ data). (d) Dithiol challenge titrations $(0.01-10 \mathrm{mM} \mathrm{DTT}, 10 \mu \mathrm{M}$ probe, $\mathrm{t}=6 \mathrm{~h}$ data $)$. (e-f) Modular probe design allows comparing cargo release kinetics. 
We next aimed to profile GSH sensitivity more meaningfully than by single-concentration assays, to reach results predictively applicable to diverse settings. Therefore, we titrated the probes with GSH over a wide concentration range ( 0.01 to $10 \mathrm{mM} \mathrm{GSH}, 10 \mu \mathrm{M}$ probe), to build logarithmic dose-response curves profiling GSH sensitivity with long-term challenge (fluorescence at $t=6 \mathrm{~h}$; Fig 6c, Fig S4-S7). These showed that linear disulfide (SS00/SS06) and 5-membered cyclic SS50-type probes are indeed quantitatively activated by physiological GSH concentrations (Fig 6c). The novel 1,2-dithiane probes however gave either zero response to GSH up to $10 \mathrm{mM}$ (SS66C, SS60), or else very low signal beginning at 100 eq GSH (SS66T; Fig 6c). As the 1,2-dithiane probes are monothiol-resistant, this indicated they might have potential for protein-selective monitoring.

We then explored a simple model system for vicinal dithiol reactivity, using DTT. Vicinal dithiols reduce cyclic disulfide triggers via a net bimolecular pathway, in contrast to monothiols (net trimolecular; Fig 2). We hoped that this mechanistic difference would allow monothiol-stable cyclic disulfide probes to be selectively activated by vicinal dithiols.

Dose-response titrations showed that the GSH-resistant SS60, SS66T and SS66C cyclic disulfide probes were indeed fully activated by DTT (Fig 6d). The linear and SS50 probes were quantitatively triggered by even equimolar DTT, and the control 056 probes were again fully resistant (Fig S4-S7). Pleasingly, the ordering of DTT resistance (SS00/SS06 < SS60 < SS66T/SS66C) was common to all cargos, again supporting the modularity of the probes' design.

As for the monothiols, we noted apparent differences of probe sensitivity to DTT depending on the cargo nature, but again we attribute this to leaving-group kinetics (PQ-OH ca. 10-fold faster-releasing than MF-OH/IG-OH; we had expected that the acidification of IG-OH would make it a faster cargo than $\mathbf{M F - O H}{ }^{75}$ but we did not see this; Fig 6e-f, Fig S4-S7). The design control probe SS60-MR had poor activation (ca. $5 \%$ at $10 \mathrm{mM}$ DTT at 6 h, Fig 6f), highlighting a general benefit of our phenol-releasing design over standard aniline-releasing probes (further detailed evaluation in the Supporting Information).

Taken together, this systematic comparison strongly showed that only 6-membered disulfides resist uncatalysed reduction by monothiols at physiological concentrations, but that such motifs can still be reduced by vicinal dithiols. To the best of our knowledge, dose-response assays evaluating e.g. GSH and DTT stability across wide concentration ranges to test for trigger reductant preferences have not been used in this field before, nor have previous reports established trigger or cargo selectivities on the basis of independently varying both triggers and cargos within the same probe series. In our opinion, both would be useful additions to the toolbox of standard assays for cumulative-release turnover probes.
2.7 Cyclic 6-membered disulfides are selectively reduced by Trx, rather than by other oxidoreductases. Having established the selectivity of the 6-membered cyclic disulfide probes for reduction by vicinal dithiols and their resistence to monothiols, we next proceeded to profile the probes' reduction by biological vicinal dithiol/disulfide-type proteins, focussing on the main components of the TrxR/Trx and GR/GSH/Grx systems. In cells, the effector proteins Trx and Grx (ca. $10 \mu \mathrm{M}$ each) have orders of magnitude higher cellular concentrations than their upstream reductases TrxR and GR (ca. $20 \mathrm{nM}$ each). To design cell-free assays to predict cellular enzyme selectivities, we ensured that the assays were performed (a) with cellular reductant concentrations, (b) using the catalytically powered redox systems rather than only pre-reduced effector proteins, and (c) examining a range of protein isoforms: both isolated from primary tissues as well as recombinantly expressed. The last point is particularly relevant for TrxR since the key selenocysteine (Sec, U) residue in its active site is highly present in isolates of native enzymes ${ }^{76}$ or recombinant forms made with novel production methodologies (up to $100 \%$ Sec contents), ${ }^{77}$ but only ca. $30 \%$ in recombinantly-expressed enzymes. ${ }^{78}$ Accordingly, for TrxR our tests included using human natively isolated TrxR1 (hTrxR1), recombinant human TrxR1, and recombinant human mitochondrial isoform TrxR2. For validation, we also wished to reference results against appropriate point mutants or analogous proteins. Thus, for TrxR, we also used the U498C mutant of human TrxR1 (hTrxR1U498C). ${ }^{13,79}$ Trx variants were examined using human natively isolated $\operatorname{Trx} 1^{80}$ (hTrx1), recombinant human Trx1, recombinant mitochondrial human Trx2, and recombinant human thioredoxin-related protein TRP $14,{ }^{81}$ which features a vicinal dithiol/disulfide-type active site that is also reduced by TrxR. Grx variants were examined using recombinant human vicinal dithiol glutaredoxins Grx1 and Grx2 together with human recombinant GR as well as human natively isolated hGR.

We evaluated all probes in fluorescence timecourses. To conclude on whether probes were reduced by the effectors Trx and Grx, and/or by direct reaction with the upstream reductants TrxR and GR, we compared assays using both effectors and upstream reductants against assays with only upstream reductants, controlling against the effectors only. We also confirmed these interpretations by titrating effectors (e.g. Trx1) in the presence of a fixed concentration of upstream reductants (e.g. TrxR1), or vice versa. This approach has to our knowledge not been reported with other compounds described as redox turnover probes, but it proved to be a powerful technique to illustrate probe selectivities. All assays were performed with $\mathrm{NADPH}$; GR assays optionally included a low concentration of GSH to allow Grx reduction without significantly reducing probe directly; see Supporting Information for further details. 


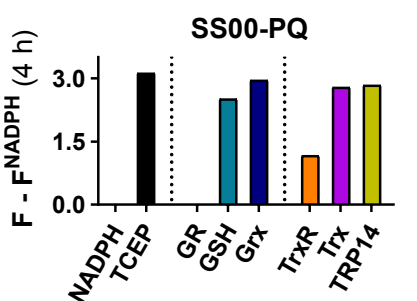

GR: - NADPH $(100 \mu \mathrm{M})+\mathrm{GR}(20 \mathrm{nM})$

GSH: $\square$ NADPH $(100 \mu \mathrm{M})+\mathrm{GR}(20 \mathrm{nM})+\mathrm{GSH}(100 \mu \mathrm{M})$

Grx: $\nabla$ NADPH $(100 \mu M)+\mathrm{GR}(20 \mathrm{nM})+\mathrm{GSH}(100 \mu \mathrm{M})+\mathrm{Grx} 1(10 \mu \mathrm{M})$
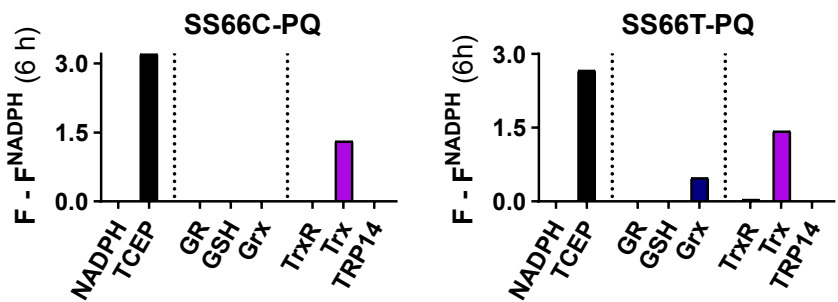

TrxR: $\neg$ NADPH $(100 \mu \mathrm{M})+\operatorname{TrxR} 1(20 \mathrm{nM})$

Trx: - NADPH $(100 \mu \mathrm{M})+\operatorname{TrxR} 1(20 \mathrm{nM})+\operatorname{Trx} 1(10 \mu \mathrm{M})$

TRP14: $-\mathrm{NADPH}(100 \mu \mathrm{M})+\operatorname{TrxR} 1(20 \mathrm{nM})+\operatorname{TRP} 14(10 \mu \mathrm{M})$

C SS60-MF: thioredoxin subtypes
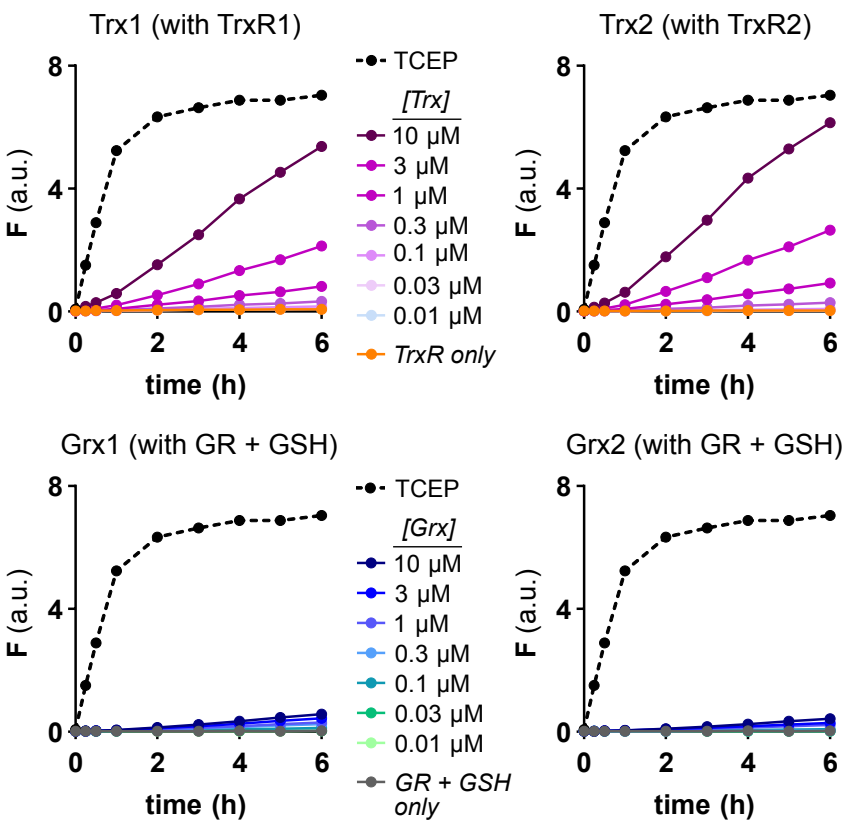

b

response kinetics
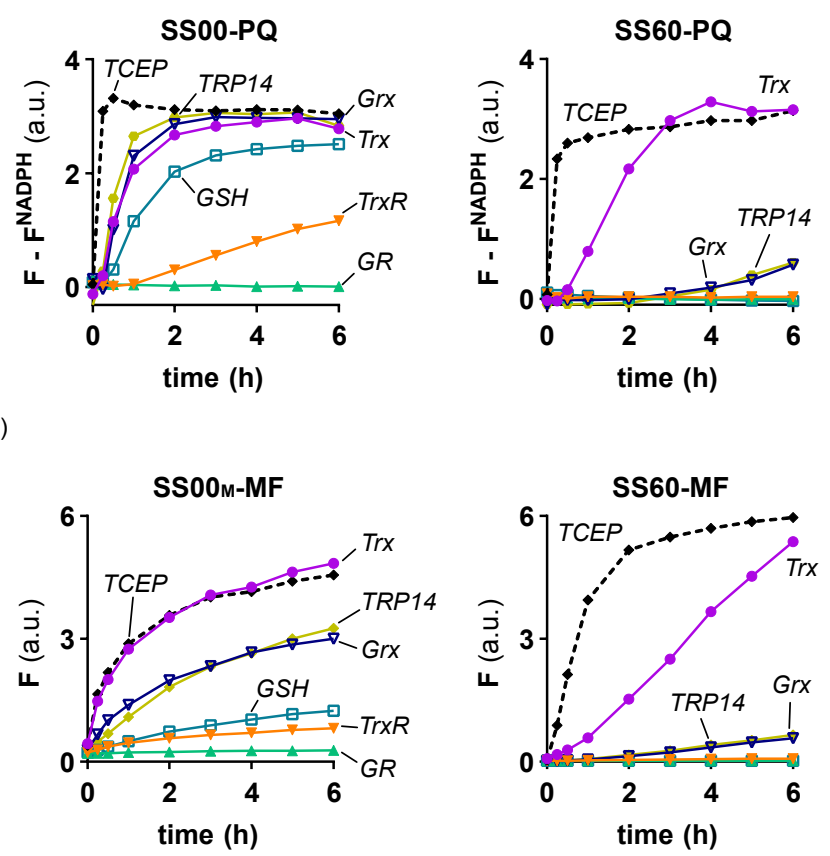

d

redox effector dose reponse (log)

SS60-MF
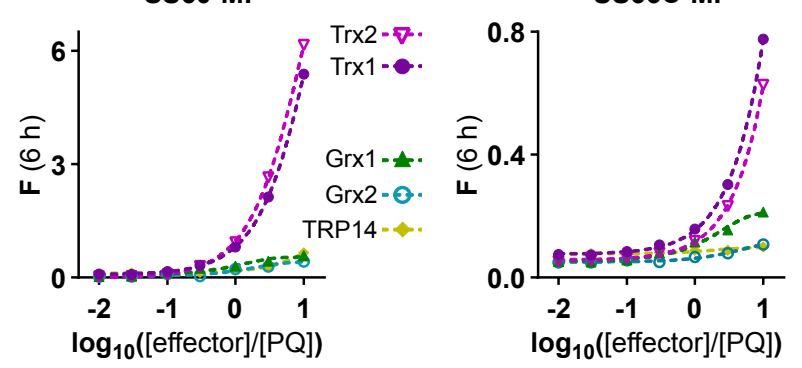

SS60-PQ
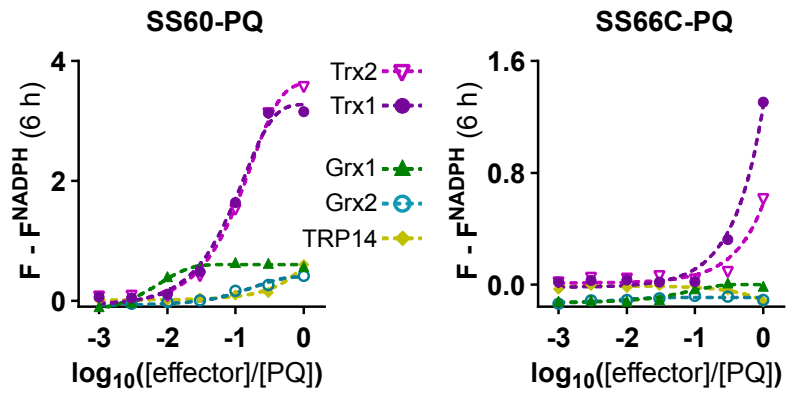

Figure 7 Cyclic 6-membered disulfides are Trx-selective substrates. (a) Bioreductant selectivity ( single-concentration profiling; $\mathrm{t}=4 \mathrm{~h}$ and $\mathrm{t}=6 \mathrm{~h}$; see also Fig S8-S9). (b) Response kinetics of SS60-PQ $(10 \mu \mathrm{M})$ and SS60-MF $(1 \mu \mathrm{M})$ compared to their linear disulfide analogues. (c) Kinetic response of SS60-MF $(1 \mu \mathrm{M})$ to titrated redox effectors Trx1, Trx2, Grx1 and Grx2. (d) Dose-response profiles of the 1,2-dithiane probes to redox effector proteins $(\mathrm{t}=6 \mathrm{~h})$. See Supporting Information for details (Fig S8-S13).

The 6-membered cyclic disulfide probes based on SS60, SS66C and SS66T were selectively activated by thioredoxins Trx1 and Trx2 (Fig 7a), with the SS60 series the most sensitive. The SS66C series had the highest Trx selectivity, with almost zero detected signal under challenge by the Grx+GSH+GR and TrxR+TRP14 cascades over 4 or 6 hours respectively (Fig 7b-c, Fig S8-S13). Both Trx1 and Trx2 dose-dependently activated the dithiane probes; though pleasingly, under relevant concentrations, these probes were non-responsive to any of the TrxR isoforms in the absence of the effector Trx. It is notable that the 1,2-dithiane-type probes were not affected by the Trx-analogue vicinal dithiol protein 
TRP14, which likely reflects the different active site structure and/or electrostatic surface charge of TRP14 compared to either Trx1 or Trx $2 .{ }^{82}$ We note that the general order of Trxselectivity in the dithiane series $($ SS66C $\gtrsim$ SS60 > SS66T) matches the order of stability against the supraphysiological monothiol levels (e.g. GSH at $10 \mathrm{mM}$, Fig 6c), supporting the earlier interpretation of structural influences on the relative kinetic instability of the trans-fused system. Matching expectations, linear disulfide reference probes $\operatorname{SSO0}(\mathbf{m})$ were activated completely non-specifically by Trx1, Trx2, TRP14, Grx1, Grx2, and TrxR1 (Fig 7a-b; Fig S8, S10, S11) in addition to the monothiols previously shown. This highlights the importance of the dithiane for achieving Trx-selectivity (Fig 2). The signal kinetics for all PQprobes were again faster than for the corresponding MF probes (Fig S8-S13).

We again performed dose-reponse titrations to test the sensitivity profiles of all probes to varying concentrations of the redox-active proteins, which should be a useful predictive tool to estimate performance in complex applications. Figure 7d shows these dose-response plots for the two major dithiane triggers, the more-Trx-sensitive SS60 and the mostTrx-selective SS66C, as applied to both PQ and MF probes (other probes in Fig S8-S13). This highlights the highly preferred processing of the novel dithiane probes by the Trx isoforms, regardless of their cargo (Fig 7d) - an excellent result for the mechanism-based design.

In summary, this systematic exploration of disulfide probe reductive triggering has relied on timecourse rather than endpoint data, and featured systematic variations and titrations of all reducing species. The results indicate that regardless of the cargos with which they are used, the hithertoreported linear and cyclic 5-membered disulfide triggers do not withstand nonselective and nonenzymatic triggering by GSH, monothiols, and a range of cellular reductants: and are therefore nonselective reduction sensors. In contrast, the new 6-membered cyclic disulfide probes display excellent selectivity and steep dose-response curves for reduction by vicinal dithiols, particularly by Trx1 and Trx2. SS60 and SS66C were thus identified as promising disulfides motifs for sensitive, modular probes selectively reduced by thioredoxins.

\section{CONCLUSIONS}

Specific dithiol-disulfide-type reactions underlie a multitude of biological pathways; and engineered disulfides are recently finding applications from chemical biology probes to biophysics and materials chemistry. Linear disulfides have been used for decades as nonspecifically and irreversibly intracellularly-cleaved substrates, ${ }^{25}$ often for intracellular release of appended cargos. While strained cyclic disulfides have found several applications, it has remained unproven whether any disulfide could be used reliably for creating robust, modular, enzyme-selective small molecule probes. ${ }^{29}$ The challenge posed has been to unite: (i) a broadly applicable, robust design that is suitable for efficiently releasing arbitrary cargos (towards both probes and prodrugs), with (ii) a disulfide/dithiol-type redox trigger that resists the high cellular background of monothiols yet can be triggered by specific redox-active dithiol-type enzymes.

In this work we used mechanism-based analysis to design novel disulfide-based trigger-cargo probes which unite both features. The 6-membered cyclic disulfide probes characterised here feature rational tuning of thermodynamic and kinetic parameters of disulfide reduction as well as of cargo release, to achieve higher stability to the monothiol background than any probes previously reported. The trigger motifs were synthesised scalably, with the novel bicyclic disulfide structures being accessed by divergent diastereomerically-pure routes, then integrated into modular phenol-releasing probes which feature stability to cleavage by non-reductive mechanisms. These probes have been evaluated through a rigorous methodology combining timecourse and dose-response screening of chemical and biological reductants. The disulfides we report, and the probe designs we apply them to, fill a gap in the literature by being the first systematic exploration of the synthesis and reductant selectivity of non-strained and non-polymerising disulfides, with the outlook towards adapting these modular phenolreleasing designs for a range of purposes. Pleasingly, the design logic of achieving selectivity by matching probe energetics and mechanism to those of the desired reducing species was supported by the selectivity in reduction of the 6-membered disulfides by the vicinal dithiol Trx, but not by the other redox active enzymes analysed here.

Further developing the Trx-selective 1,2-dithiane chemotypes for cellular monitoring of endogenous Trx is a major aim of our ongoing research. This faces challenges including (1) ensuring reduction is competitive with native Trx substrates, to reach high turnover or signal in the cellular context; and (2) favouring the post-reduction cyclisation $k_{\text {rel }}$ relative to the re-oxidation pathways (particularly $\mathrm{k}_{\mathrm{g} 2}$; Fig 2) to maximise the proportion of Trx-reduced probe which releases its cargo. These are nontrivial challenges. We also expect that tuning the probes' intracellular localisation will prove crucial for performance.

Diversifying disulfide trigger structures to address other key oxidoreductases is another goal of our research. The scope of redox biology would be greatly expanded by developing probes that are GSSG-competitive substrates of GR, or Trxcompetitive substrates of TrxR. This would allow researchers to tap into the turnover dynamics that drive the major dithiol/disulfide-manifold processes throughout the cell. This work has demonstrated that disulfides are a stilluntapped well of potential for redox probes. We therefore hope that further developments of probes with increased selectivity for specific redox enzymes will facilitate progress in the many areas and applications of research in redox biology. 


\section{ASSOCIATED CONTENT}

\section{Supporting Information}

The Supporting Information is available free of charge at DOI xxxx:

Synthesis, analysis, and biochemistry (PDF)

\section{AUTHOR INFORMATION}

\section{Corresponding Author}

* oliver.thorn-seshold@cup.Imu.de

ORCIDs

Jan G. Felber: 0000-0002-5010-9624

Julia Thorn-Seshold: 0000-0002-4879-4159

Oliver Thorn-Seshold: 0000-0003-3981-651X

\section{Author Contributions}

J.G.F. performed synthesis, analysis, chemoreductant and enzyme studies, coordinated data assembly and wrote the manuscript. L.Z. performed synthesis, analysis, and chemoreductant assays. L.P. and K.S. performed enzyme titration assays. S.B. and C.B. performed enzyme selectivity screenings. M.M. performed synthesis and analysis. U.T. performed early biological evaluations. K.B., E.S.J.A. and J.T.-S. supervised enzymatic selectivity screenings. O.T.-S. designed the concept and experiments, supervised all other experiments, coordinated data assembly and wrote the manuscript, with input from all co-authors.

\section{Notes}

J.F., L.Z., J.T.-S. and O.T.-S. are inventors on a patent application filed by the LMU Munich in 2021 covering compound structures reported in this paper. The authors declare no competing financial interest.

\section{ACKNOWLEDGMENT}

This research was supported by funds from the German Research Foundation (DFG: SFB 1032 project B09 number 201269156, SFB TRR 152 project P24 number 239283807, SPP 1926 project number 426018126, and Emmy Noether grant 400324123 to O.T.-S.; SPP 1710 project BE 1540/23-2 to K.B. (now transferred to Stefan Rahlfs)); LMUExcellent (Junior Researcher Fund to O.T.-S.); the Munich Centre for NanoScience initiative (CeNS) to O.T.-S.; and from Karolinska Institutet, The Knut and Alice Wallenberg Foundations, The Swedish Cancer Society, The Swedish Research Council and the Hungarian Thematic Excellence Programme (TKP2020-NKA-26) to E.S.J.A.. J.G.F. thanks the Studienstiftung des deutschen Volkes for support through a PhD scholarship; L.Z. thanks the Fonds der Chemischen Industrie for support through a PhD scholarship; L.P. thanks the GRK 2338 for support through a PhD scholarship; J.T.-S. thanks the Joachim Herz Foundation for fellowship support. We thank Peter Mayer (LMU) for X-ray crystallography; Qing Cheng (KI) for production and purification of recombinant enzymes; Julia Rauh (LMU) for synthetic assistance; and Matt Fuchter (ICL), Klaus T. Wanner (LMU), Kate Carroll (Scripps), and the attendees of the SPP 1710 conference Thiol-Based Redox Switches in 2019, for their supportive and collegial discussions.

This paper is dedicated to George Whitesides, who so greatly contributed to setting thiol/disulfide redox chemistry on secure theoretical and practical foundations.

\section{REFERENCES}

(1) Herrmann, J. M.; Dick, T. P. Redox Biology on the Rise. Biol Chem 2012, 393 (9), 999-1004. https://doi.org/10.1515/hsz-2012-0111.

(2) Jones, D. P.; Sies, H. The Redox Code. ARS 2015, 23 (9), 734-746. https://doi.org/10.1089/ars.2015.6247.

(3) Arnér, E. S. J.; Holmgren, A. Physiological Functions of Thioredoxin and Thioredoxin Reductase. Eur J Biochem 2000, 267 (20), 6102-6109. https://doi.org/10.1046/j.1432-1327.2000.01701.x.

(4) Miller, C. G.; Holmgren, A.; Arnér, E. S. J.; Schmidt, E. E. NADPHDependent and -Independent Disulfide Reductase Systems. Free Radical Biology Medicine 2018, 127, 248-261. https://doi.org/10.1016/j.freeradbiomed.2018.03.051.

(5) Pillay, C. S.; Hofmeyr, J.-H. S.; Rohwer, J. M. The Logic of Kinetic Regulation in the Thioredoxin System. BMC Systems Biology 2011, 5 (1), 15. https://doi.org/10.1186/1752-0509-5-15.

(6) Deponte, M. Glutathione Catalysis and the Reaction Mechanisms of Glutathione-Dependent Enzymes. Biochimica Biophysica Acta BBA - General Subjects 2013, $1830 \quad$ (5), 3217-3266. https://doi.org/10.1016/j.bbagen.2012.09.018.

(7) Lu, J.; Holmgren, A. The Thioredoxin Antioxidant System. Free Radical Biology Medicine 2014, 66, 75-87. https://doi.org/10.1016/j.freeradbiomed.2013.07.036.

(8) Fernandes, A. P.; Holmgren, A. Glutaredoxins: GlutathioneDependent Redox Enzymes with Functions Far Beyond a Simple Thioredoxin Backup System. ARS 2004, 6 (1), 63-74. https://doi.org/10.1089/152308604771978354.

(9) Holmgren, A.; Johansson, C.; Berndt, C.; Lönn, M. E.; Hudemann, C.; Lillig, C. H. Thiol Redox Control via Thioredoxin and Glutaredoxin Systems. Biochemical Society Transactions 2005, 33 (6), 1375 https://doi.org/10.1042/BST20051375.

(10) Hanschmann, E.-M.; Godoy, J. R.; Berndt, C.; Hudemann, C.; Lillig, C. H. Thioredoxins, Glutaredoxins, and Peroxiredoxins-Molecular Mechanisms and Health Significance: From Cofactors to Antioxidants to Redox Signaling. ARS 2013, 19 (13), 1539-1605. https://doi.org/10.1089/ars.2012.4599.

(11) Fridovich, I.; Poole, L. B.; Holmgren, A.; Lou, M. F.; Gladyshev, V. N.; David, S. S.; Osborne, R. L.; Dawson, J. H.; Copley, S. D.; Kadokura, H.; Beckwith, J.; Gilbert, H. F.; Ragsdale, S. W. Antioxidant Enzymes. In Redox Biochemistry; Wiley Online Books; Wiley, 2007; pp 49-134. https://doi.org/10.1002/9780470177334.ch3.

(12) Cheng, Q.; Sandalova, T.; Lindqvist, Y.; Arnér, E. S. J. Crystal Structure and Catalysis of the Selenoprotein Thioredoxin Reductase 1. J Biol Chem 2009, 284 (6), 3998-4008. https://doi.org/10.1074/jbc.M807068200.

(13) Fritz-Wolf, K.; Urig, S.; Becker, K. The Structure of Human Thioredoxin Reductase 1 Provides Insights into C-Terminal Rearrangements During Catalysis. Journal Of Molecular Biology 2007, 370 (1), 116-127. https://doi.org/10.1016/j.jmb.2007.04.044.

(14) Gromer, S.; Johansson, L.; Bauer, H.; Arscott, L. D.; Rauch, S.; Ballou, D. P.; Williams, C. H.; Schirmer, R. H.; Arner, E. S. J. Active Sites of Thioredoxin Reductases: Why Selenoproteins? Proceedings Of National Academy Of Sciences 2003, $100 \quad$ (22), $12618-12623$. https://doi.org/10.1073/pnas.2134510100.

(15) Arnér, E. S. J. Focus on Mammalian Thioredoxin Reductases Important Selenoproteins with Versatile Functions. Biochimica Biophysica Acta BBA - General Subjects 2009, $1790 \quad$ (6), 495-526. https://doi.org/10.1016/j.bbagen.2009.01.014.

(16) Maroney, M. J.; Hondal, R. J. Selenium versus Sulfur: Reversibility of Chemical Reactions and Resistance to Permanent Oxidation in Proteins and 
Nucleic Acids. Selenium Research In Redox Biology Health Disease - 200 Years $\begin{array}{lllll}\text { Anniversary Issue 2018, 228-237. } & \text { 201, }\end{array}$ https://doi.org/10.1016/j.freeradbiomed.2018.03.035.

(17) Sagemark, J.; Elgán, T. H.; Bürglin, T. R.; Johansson, C.; Holmgren, A.; Berndt, K. D. Redox Properties and Evolution of Human Glutaredoxins. Proteins Structure Function Bioinformatics 2007, 68 (4), 879-892. https://doi.org/10.1002/prot.21416.

(18) Weichsel, A.; Gasdaska, J. R.; Powis, G.; Montfort, W. R. Crystal Structures of Reduced, Oxidized, and Mutated Human Thioredoxins: Evidence for a Regulatory Homodimer. Structure 1996, 4 (6), 735-751. https://doi.org/10.1016/S0969-2126(96)00079-2.

(19) Johansson, L.; Arscott, L. D.; Ballou, D. P.; Williams Jr., C. H.; Arnér, E. S. J. Studies of an Active Site Mutant of the Selenoprotein Thioredoxin Reductase: The Ser-Cys-Cys-Ser Motif of the Insect Orthologue Is Not Sufficient to Replace the Cys-Sec Dyad in the Mammalian Enzyme. Free Radical Biology Medicine 2006, 41 (4), 649-656. https://doi.org/10.1016/j.freeradbiomed.2006.05.005.

(20) Pan, J. L.; Bardwell, J. C. A. The Origami of Thioredoxin-like Folds. Protein Science 2006, 15 (10), 2217-2227. https://doi.org/10.1110/ps.062268106.

(21) Palde, P. B.; Carroll, K. S. A Universal Entropy-Driven Mechanism for Thioredoxin-Target Recognition. Proceedings Of National Academy Of Sciences 2015, 112 (26), 7960. https:// doi.org/10.1073/pnas.1504376112.

(22) Meyer, A. J.; Dick, T. P. Fluorescent Protein-Based Redox Probes. ARS 2010, 13 (5), 621-650. https://doi.org/10.1089/ars.2009.2948.

(23) Lukyanov, K. A.; Belousov, V. V. Genetically Encoded Fluorescent Redox Sensors. Biochimica Biophysica Acta BBA - General Subjects 2014, 1840 (2), 745-756. https://doi.org/10.1016/j.bbagen.2013.05.030.

(24) Schwarzländer, M.; Dick, T. P.; Meyer, A. J.; Morgan, B. Dissecting Redox Biology Using Fluorescent Protein Sensors. ARS 2016, 24 (13), 680-712. https://doi.org/10.1089/ars.2015.6266.

(25) Lee, M. H.; Yang, Z.; Lim, C. W.; Lee, Y. H.; Dongbang, S.; Kang, C.; Kim, J. S. Disulfide-Cleavage-Triggered Chemosensors and Their Biological Applications. Chemical Reviews 2013, 113 (7), 5071-5109. https://doi.org/10.1021/cr300358b.

(26) Pires, M. M.; Chmielewski, J. Fluorescence Imaging of Cellular Glutathione Using a Latent Rhodamine. OL 2008, 10 (5), 837-840. https://doi.org/10.1021/ol702769n.

(27) Lim, C. S.; Masanta, G.; Kim, H. J.; Han, J. H.; Kim, H. M.; Cho, B. R. Ratiometric Detection of Mitochondrial Thiols with a Two-Photon Fluorescent Probe. JACS 2011, 133 (29), 11132-11135. https://doi.org/10.1021/ja205081s.

(28) Butora, G.; Qi, N.; Fu, W.; Nguyen, T.; Huang, H.-C.; Davies, I. W. Cyclic-Disulfide-Based Prodrugs for Cytosol-Specific Drug Delivery. Angewandte Chemie International Edition 2014, 53 (51), 14046-14050. https://doi.org/10.1002/anie.201407130.

(29) Felber, J.; Poczka, L.; Busker, S.; Theisen, U.; Zeisel, L.; Maier, M.; Loy, K.; Brandstädter, C.; Becker, K.; Arner, E.; Thorn-Seshold, J.; ThornSeshold, O. Cyclic 5-Membered Disulfides Are Not Selective Substrates of Thioredoxin Reductase, but Are Opened Nonspecifically by Thiols. ChemRxiv 2020. https://doi.org/10.26434/chemrxiv.13483155.v1.

(30) Cherblanc, F. L.; Chapman, K. L.; Brown, R.; Fuchter, M. J. Chaetocin Is a Nonspecific Inhibitor of Histone Lysine Methyltransferases. NChB 2013, 9 (3), 136-137. https://doi.org/10.1038/nchembio.1187.

(31) Zhang, L.; Duan, D.; Liu, Y.; Ge, C.; Cui, X.; Sun, J.; Fang, J. Highly Selective Off-On Fluorescent Probe for Imaging Thioredoxin Reductase in Living Cells. JACS 2014, $136 \quad$ (1), 226-233. https://doi.org/10.1021/ja408792k.

(32) Ma, H.; Zhang, J.; Zhang, Z.; Liu, Y.; Fang, J. A Fast Response and Red Emission Probe for Mammalian Thioredoxin Reductase. Chemical Communications 2016, 52 (81), 12060-12063. https://doi.org/10.1039/C6CC04984B.

(33) Li, X.; Zhang, B.; Yan, C.; Li, J.; Wang, S.; Wei, X.; Jiang, X.; Zhou, P.; Fang, J. A Fast and Specific Fluorescent Probe for Thioredoxin Reductase That Works via Disulphide Bond Cleavage. NComm 2019, 10 (2745), 1-12. https://doi.org/10.1038/s41467-019-10807-8.

(34) Li, X.; Hou, Y.; Meng, X.; Ge, C.; Ma, H.; Li, J.; Fang, J. Selective Activation of a Prodrug by Thioredoxin Reductase Providing a Strategy to Target Cancer Cells. Angewandte Chemie International Edition 2018, 57 (21), 61416145. https://doi.org/10.1002/anie.201801058.
(35) Houk, J.; Whitesides, G. M. Structure-Reactivity Relations for ThiolDisulfide Interchange. JACS 1987, 109 (22), 6825-6836. https://doi.org/10.1021/ja00256a040.

(36) Singh, R.; Whitesides, G. M. Degenerate Intermolecular ThiolateDisulfide Interchange Involving Cyclic Five-Membered Disulfides Is Faster by Approx. $10^{3}$ than That Involving Six- or Seven-Membered Disulfides. JACS 1990, 112 (17), 6304-6309. https://doi.org/10.1021/ja00173a018.

(37) Abegg, D.; Gasparini, G.; Hoch, D. G.; Shuster, A.; Bartolami, E.; Matile, S.; Adibekian, A. Strained Cyclic Disulfides Enable Cellular Uptake by Reacting with the Transferrin Receptor. JACS 2017, 139 (1), 231-238. https://doi.org/10.1021/jacs.6b09643.

(38) Gasparini, G.; Sargsyan, G.; Bang, E.-K.; Sakai, N.; Matile, S. Ring Tension Applied to Thiol-Mediated Cellular Uptake. Angewandte Chemie International Edition 2015, $54 \quad$ (25), 7328-7331. https://doi.org/10.1002/anie.201502358.

(39) Butora, G. Nucleoside Kinase Bypass Compositions and Methods. WO2014088923A1, 2014.

(40) Hayashi, J.; Samezawa, Y.; Ochi, Y.; Wada, S.; Urata, H. Syntheses of Prodrug-Type Phosphotriester Oligonucleotides Responsive to Intracellular Reducing Environment for Improvement of Cell Membrane Permeability and Nuclease Resistance. $B M C L \quad \mathbf{2 0 1 7}, 27$ (14), 3135-3138. https://doi.org/10.1016/j.bmcl.2017.05.031.

(41) Kong, F.; Zhao, Y.; Liang, Z.; Liu, X.; Pan, X.; Luan, D.; Xu, K.; Tang, B. Highly Selective Fluorescent Probe for Imaging $\mathrm{H}_{2} \mathrm{Se}$ in Living Cells and in Vivo Based on the Disulfide Bond. Analytical Chemistry 2017, 89 (1), 688-693. https://doi.org/10.1021/acs.analchem.6b03136.

(42) Ziv, I. Compounds and Methods for Trans-Membrane Delivery of Molecules. US9687556B2, 2017.

(43) Ziv, I. Pro-Drugs and Related Methods. WO2017017669A1, 2017.

(44) Na, Y.; Kohn, H. Quinone-Cyclized Porfiromycins. Heterocycles 2001, 55 (7), 1347-1364. https://doi.org/10.3987/COM-01-9215.

(45) Lee, S. H.; Kohn, H. Cyclic Disulfide C(8) Iminoporfiromycin: Nucleophilic Activation of a Porfiromycin. JACS 2004, 126 (13), 4281-4292. https:// doi.org/10.1021/ja030577r.

(46) Ollivier, N.; Dheur, J.; Mhidia, R.; Blanpain, A.; Melnyk, O. Bis(2Sulfanylethyl)Amino Native Peptide Ligation. OL 2010, 12 (22), 5238-5241. https://doi.org/10.1021/ol102273u.

(47) Zhu, B.; Zhang, X.; Li, Y.; Wang, P.; Zhang, H.; Zhuang, X. A Colorimetric and Ratiometric Fluorescent Probe for Thiols and Its Bioimaging Applications. Chemical Communications 2010, 46 (31), 5710. https://doi.org/10.1039/c0cc00477d.

(48) Hong, K.-H.; Kim, D. I.; Kwon, H.; Kim, H.-J. A Fluoresceinylcarbonate-Based Fluorescent Probe for the Sensitive Detection of Biothiols in a HEPES Buffer and Its Cellular Expression. RSC Advances 2014, 4 (2), 978-982. https://doi.org/10.1039/C3RA42935K.

(49) Dan, K.; Veetil, A. T.; Chakraborty, K.; Krishnan, Y. DNA Nanodevices Map Enzymatic Activity in Organelles. Nature Nanotechnology 2019, 14 (3), 252-259. https://doi.org/10.1038/s41565-019-0365-6.

(50) Mata, G.; do Rosário, V. E.; Iley, J.; Constantino, L.; Moreira, R. A Carbamate-Based Approach to Primaquine Prodrugs: Antimalarial Activity, Chemical Stability and Enzymatic Activation. Chemical Proteomics 2012, 20 (2), 886-892. https://doi.org/10.1016/j.bmc.2011.11.059.

(51) Ghosh, A. K.; Brindisi, M. Organic Carbamates in Drug Design and Medicinal Chemistry. JMC 2015, 58 (7), 2895-2940. https://doi.org/10.1021/jm501371s.

(52) Vacondio, F.; Silva, C.; Mor, M.; Testa, B. Qualitative StructureMetabolism Relationships in the Hydrolysis of Carbamates. Drug Metabolism Reviews 2010, 42 (4), 551-589. https://doi.org/10.3109/03602531003745960.

(53) Thorn-Seshold, O.; Vargas-Sanchez, M.; McKeon, S.; Hasserodt, J. A Robust, High-Sensitivity Stealth Probe for Peptidases. Chemical $\begin{array}{llll}\text { Communications 2012, } & 48 \text { (50), 6253-6255. }\end{array}$ https://doi.org/10.1039/C2CC32227G.

(54) Tian, L.; Yang, Y.; Wysocki, L. M.; Arnold, A. C.; Hu, A.; Ravichandran, B.; Sternson, S. M.; Looger, L. L.; Lavis, L. D. Selective EsteraseEster Pair for Targeting Small Molecules with Cellular Specificity. Proceedings of National Academy of Sciences 2012, 109 (13), 4756-4761. https://doi.org/10.1073/pnas.1111943109.

(55) Orte, A.; Crovetto, L.; Talavera, E. M.; Boens, N.; Alvarez-Pez, J. M. Absorption and Emission Study of 2', $7^{\prime}$-Difluorofluorescein and Its Excited-State 
Buffer-Mediated Proton Exchange Reactions. JPC-A 2005, 109 (5), 734-747. https://doi.org/10.1021/jp046786v.

(56) Machida, T.; Dutt, S.; Winssinger, N. Allosterically Regulated Phosphatase Activity from Peptide-PNA Conjugates Folded Through Hybridization. Angewandte Chemie International Edition 2016, 55 (30), 85958598. https://doi.org/10.1002/anie.201602751.

(57) Rando, G.; Winssinger, N.; Lindberg, E.; Anzola, M. New Compounds and Uses Thereof for Detection of Target Molecules in a Sample. US $2019 / 0315713 \mathrm{~A} 1$.

(58) Fu, Q.; Li, H.; Duan, D.; Wang, C.; Shen, S.; Ma, H.; Liu, Z. ExternalRadiation-Induced Local Hydroxylation Enables Remote Release of Functional Molecules in Tumors. Angewandte Chemie International Edition 2020, 59 (48), 21546-21552. https://doi.org/10.1002/anie.202005612.

(59) Lukesh, J. C.; Palte, M. J.; Raines, R. T. A Potent, Versatile DisulfideReducing Agent from Aspartic Acid. JACS 2012, 134 (9), 4057-4059. https://doi.org/10.1021/ja211931f.

(60) Lees, W. J.; Whitesides, G. M. Equilibrium Constants for ThiolDisulfide Interchange Reactions: A Coherent, Corrected Set. JOC 1993, 58 (3), 642-647. https://doi.org/10.1021/jo00055a016.

(61) Chênevert, R.; Dickman, M. Enzymatic Route to Chiral, Nonracemic Cis-2,6- and Cis,Cis-2,4,6-Substituted Piperidines. Synthesis of $(+)$ Dihydropinidine and Dendrobate Alkaloid (+)-241D. JOC 1996, 61 (10), 33323341. https://doi.org/10.1021/jo9519569.

(62) Corre, L. L.; Kizirian, J.-C.; Levraud, C.; Boucher, J.-L.; Bonnet, V.; Dhimane, H. Diastereoselective Functionalizations of Enecarbamates Derived from Pipecolic Acid towards 5-Guanidinopipecolates as Arginine Mimetics. $O B C$ 2008, 6 (18), 3388-3398. https://doi.org/10.1039/B805811C.

(63) Vartak, A. P.; Gabriela Deaciuc, A.; Dwoskin, L. P.; Crooks, P. A. Quinlobelane: A Water-Soluble Lobelane Analogue and Inhibitor of VMAT2. $\begin{array}{lllll}B M C L & 2010, & 20 & \text { (12), }\end{array}$ https://doi.org/10.1016/j.bmcl.2010.04.117.

(64) Yang, B. V.; O’Rourke, D.; Li, J. Mild and Selective Debenzylation of Tertiary Amines Using $\alpha$-Chloroethyl Chloroformate. Synlett 2002, 1993 (03), 195-196. https://doi.org/10.1055/s-1993-22398.

(65) Capasso, S.; Zagari, A. 1,2-Dithiane-4,5-Diol. Acta Crystallographica Section B Structural Crystallography Crystal Chemistry 1981, 37 (7), 1437-1439. https://doi.org/10.1107/S0567740881006195.

(66) Kilgore, H. R.; Raines, R. T. Disulfide Chromophores Arise from Stereoelectronic Effects. JPC-B 2020, 124 (19), 3931-3935. https://doi.org/10.1021/acs.jpcb.0c02272.

(67) Burns, J. A.; Whitesides, G. M. Predicting the Stability of Cyclic Disulfides by Molecular Modeling: Effective Concentrations in Thiol-Disulfide Interchange and the Design of Strongly Reducing Dithiols. JACS 1990, 112 (17), 6296-6303. https://doi.org/10.1021/ja00173a017.

(68) Lüttringhaus, A.; Brechlin, A. Cyclische Disulfide, III.cis- undtrans2.3-Dithia-decalin. Chem Ber 1959, 92 (9), 2271-2277. https://doi.org/10.1002/cber.19590920945.

(69) Rothwarf, D. M.; Scheraga, H. A. Equilibrium and Kinetic Constants for the Thiol-Disulfide Interchange Reaction between Glutathione and Dithiothreitol. Proceedings Of National Academy Of Sciences 1992, 89 (17), 7944 7948. https://doi.org/10.1073/pnas.89.17.7944.

(70) Fridovich, I.; Poole, L. B.; Holmgren, A.; Lou, M. F.; David, S. S.; Osborne, R. L.; Dawson, J. H.; Copley, S. D.; Kadokura, H.; Beckwith, J.; Gilbert, H. F.; Ragsdale, S. W.; Gladyshev, V. N.; Becker, D.; Dickmann, M.; Banerjee, R. Antioxidant Enzymes. In Antioxidant Enzymes; Redox Biochemistry; John Wiley \& Sons, Inc., 2007; pp 49-134.

(71) Jones, D. P. Protein Sensors and Reactive Oxygen Species. In Redox Potential of GSH/GSSG Couple: Assay and Biological Significance; Methods in Enzymology; Academic Press, 2002; pp 93-112.

(72) Jia, H.; Hu, G.; Shi, D.; Gan, L.; Zhang, H.; Yao, X.; Fang, J. Fluorophore-Dependent Cleavage of Disulfide Bond Leading to a Highly Selective Fluorescent Probe of Thioredoxin. Analytical Chemistry 2019, 91 (13), 8524-8531. https://doi.org/10.1021/acs.analchem.9b01779.

(73) Dal Corso, A.; Borlandelli, V.; Corno, C.; Perego, P.; Belvisi, L.; Pignataro, L.; Gennari, C. Fast Cyclization of a Proline-Derived Self-Immolative Spacer Improves the Efficacy of Carbamate Prodrugs. Angewandte Chemie International Edition 2020, 59 (10), 4176-4181. https://doi.org/10.1002/anie.201916394.

(74) Laurent, Q.; Sakai, N.; Matile, S. The Opening of 1,2-Dithiolanes and 1,2-Diselenolanes: Regioselectivity, Rearrangements, and Consequences for
Poly(Disulfide)s, Cellular Uptake and Pyruvate Dehydrogenase Complexes. Helv Chim 2019, 102 (2), e1800209. https://doi.org/10.1002/hlca.201800209.

(75) Chyan, W.; Kilgore, H. R.; Gold, B.; Raines, R. T. Electronic and Steric Optimization of Fluorogenic Probes for Biomolecular Imaging. JOC 2017, 82 (8), 4297-4304. https://doi.org/10.1021/acs.joc.7b00285.

(76) Cheng, Q.; Arnér, E. S. J. Selenocysteine Insertion at a Predefined UAG Codon in a Release Factor 1 (RF1)-Depleted Escherichia Coli Host Strain Bypasses Species Barriers in Recombinant Selenoprotein Translation. J Biol Chem 2017, 292 (13), 5476-5487. https://doi.org/10.1074/jbc.M117.776310.

(77) Cheng, Q.; Arnér, E. S. J. Overexpression of Recombinant Selenoproteins in E. Coli. In Selenoproteins; Chavatte, L., Ed.; Methods in Molecular Biology; Springer New York: New York, NY, 2018; Vol. 1661, pp 231 240. https://doi.org/10.1007/978-1-4939-7258-6_17.

(78) Lacey, B. M.; Eckenroth, B. E.; Flemer, S.; Hondal, R. J. Selenium in Thioredoxin Reductase: A Mechanistic Perspective. Biochem 2008, 47 (48), 12810-12821. https://doi.org/10.1021/bi800951f.

(79) Fritz-Wolf, K.; Kehr, S.; Stumpf, M.; Rahlfs, S.; Becker, K. Crystal Structure of the Human Thioredoxin Reductase-Thioredoxin Complex. NComm 2011, 2 (383), 1-8. https://doi.org/10.1038/ncomms1382.

(80) Gromer, S.; Arscott, L. D.; Williams, C. H.; Schirmer, R. H.; Becker, K. Human Placenta Thioredoxin Reductase: Isolation of the Selenoenzmye, Steady State Kinetics, and Inhibition by Therapeutic Gold Compounds. J Biol $\begin{array}{lllll}\text { Chem } & 1998, & 273 & \text { (32), 20096-20101. }\end{array}$ https://doi.org/10.1074/jbc.273.32.20096.

(81) Espinosa, B.; Arnér, E. S. J. Thioredoxin-Related Protein of $14 \mathrm{KDa}$ as a Modulator of Redox Signalling Pathways: TRP14 in Redox Signalling. $\mathrm{Br} J$ Pharmacol 2019, 176 (4), 544-553. https://doi.org/10.1111/bph.14479.

(82) Woo, J. R.; Kim, S. J.; Jeong, W.; Cho, Y. H.; Lee, S. C.; Chung, Y. J.; Rhee, S. G.; Ryu, S. E. Structural Basis of Cellular Redox Regulation by Human TRP14. J Biol Chem 2004, 279 (46), 48120-48125. https://doi.org/10.1074/jbc.M407079200. 


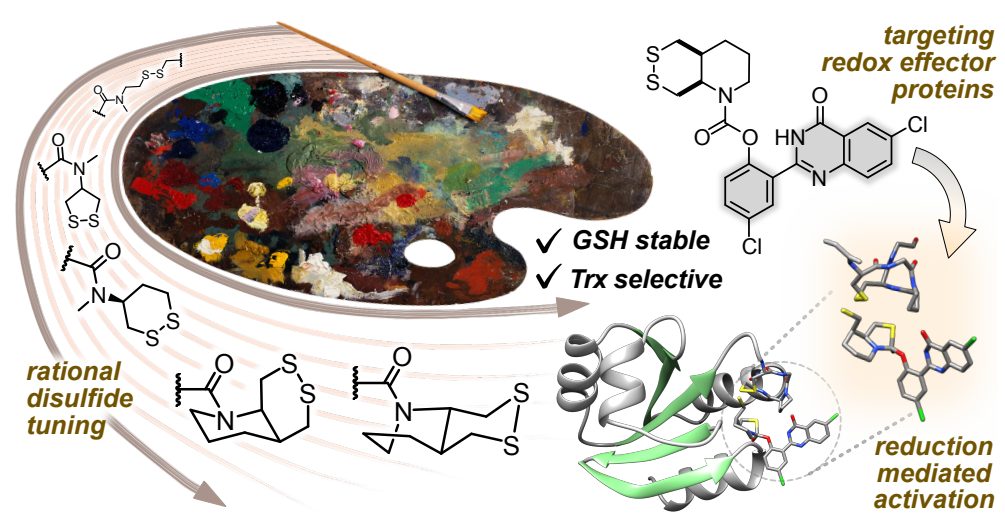

